\title{
DECOMPOSITION MATRICES FOR EXCEPTIONAL GROUPS AT $d=4$
}

\author{
OLIVIER DUDAS AND GUNTER MALLE
}

\begin{abstract}
We determine the decomposition matrices of unipotent $\ell$-blocks of defect $\Phi_{4}^{2}$ for exceptional groups of Lie type up to a few unknowns. For this we employ the new cohomological methods of the first author, together with properties of generalized Gelfand-Graev characters which were recently shown to hold whenever the underlying characteristic is good.
\end{abstract}

\section{IntRODUCTION}

The main aim of this paper is to determine the decomposition matrices for the unipotent blocks of finite exceptional groups of Lie type $E_{6}(q),{ }^{2} E_{6}(q), E_{7}(q), E_{8}(q)$ and $F_{4}(q)$ for odd primes $\ell$ dividing $\Phi_{4}(q)=q^{2}+1$. For these groups, we study only the blocks with defect at most 2 - which amounts to excluding only the principal block of $E_{8}(q)$ - and we obtain an approximation to the decomposition matrices in that a small number of entries remain undetermined for ${ }^{2} E_{6}(q)$ and $F_{4}(q)$. On the way, we also find decomposition matrices for orthogonal groups of rank up to 7 which occur as Levi subgroups. As a byproduct, we obtain the repartition of the simple unipotent modules into Harish-Chandra series.

The decomposition matrices are determined inductively, by the combination of standard methods like Harish-Chandra induction and restriction, and the new ingredient introduced in [9] to tackle the discrete series. Our strategy can be summarized in the following three steps.

Step 1. We start by using Harish-Chandra induction from proper Levi subgroups, HarishChandra restriction from suitable overgroups and decomposition numbers for Hecke algebras to compute the columns of the decomposition matrix corresponding to the non-cuspidal simple modules. In several cases this determines entirely the unipotent part of the decomposition matrix.

Step 2. We consider suitable generalized Gelfand-Graev characters containing the missing columns. The properties of these projective characters (see [20, 23]) force the decomposition matrix to be unitriangular, but their construction introduce some conditions on the underlying characteristic of the groups considered.

Step 3. Finally, we use virtual projective characters afforded by cohomology of DeligneLusztig varieties. As observed in [9], we have some control on the multiplicity of the various PIMs in these virtual characters, from which we deduce upper bounds on the

Date: September 19, 2021.

2010 Mathematics Subject Classification. Primary 20C33; Secondary 20G40.

The second author gratefully acknowledges financial support by ERC Advanced Grant 291512. 
missing decomposition numbers. In many cases these bounds are small enough to determine the numbers.

The paper is built up as follows. In Section 2 we present the general methods used in many of the arguments. Then in Section 3 we determine decomposition matrices for some orthogonal groups of type $D_{n}, n \leq 8$. In the following sections, we consider the exceptional groups of type $E_{n}, 6 \leq n \leq 8$. For these we are able to determine all the decomposition numbers for blocks of defect at most 2 , which excludes only the principal block of groups of types $D_{7}, D_{8}$ and $E_{8}$. In Section 7 we turn to the twisted groups of types ${ }^{2} D_{n}, n \leq 7$, and ${ }^{2} E_{6}$. We finish by the case of symplectic groups of type $C_{n}$, $n \leq 4$ and exceptional groups of type $F_{4}$ in Section 8. In those cases we have to assume that the underlying characteristic is good, and even then we are not able to determine all decomposition numbers. Some entries remain unknown, but we still give conditions and relations that they satisfy.

Let us note that our calculations give rather large examples for Geck's conjectures on the shape of $\ell$-decomposition matrices (see [12, Conjecture 3.4] for a precise formulation of the conjectures). Indeed, in all the cases we consider we observe that:

- the decomposition matrix has a unitriangular shape, with respect to an order compatible with Lusztig's a-function,

- within a given family, the square submatrix is the identity matrix (up to some indeterminates for types $D_{7},{ }^{2} D_{7},{ }^{2} E_{6}$ and $C_{4}$ ),

- any cuspidal unipotent character remains irreducible after $\ell$-reduction (more generally any unipotent character with smallest $a$-function within its Harish-Chandra series).

\section{Methods}

We determine decomposition matrices for unipotent blocks of various families of groups of Lie type $G$, where $G=\mathbf{G}(q)$ is the group of fixed points under a Frobenius endomorphism with respect to an $\mathbb{F}_{q}$-structure of a simple algebraic group $\mathbf{G}$ over the algebraic closure of $\mathbb{F}_{q}$. More precisely, we consider the case that $\ell$ is an odd prime dividing $q^{2}+1$. In particular, we have $\ell \geq 5$ always. In the proofs we make use of several standard arguments which we collect here for easier reference.

Firstly, the subdivision of unipotent characters into $\ell$-blocks is known in our situation, see [3]. Secondly, by results of Geck and Hiss, whenever $\ell$ is a good prime for the group in question, then the unipotent characters form a basic set for the union of unipotent blocks. Thus, for good primes the decomposition matrix for a unipotent block is known once the decomposition numbers for the unipotent characters in that block have been found. To determine the decomposition matrix of the block is hence equivalent to finding the (unipotent parts of the) ordinary characters of all projective indecomposable modules (PIMs) in that block.

One standard method for constructing projective characters is via Harish-Chandra induction $R_{L}^{G}$ of projective characters from proper Levi subgroups $L$, which we may assume to be know by induction. Thus our first source for projective characters is

(HCi) Harish-Chandra induction of projective characters from proper Levi subgroups. 
This Harish-Chandra induction can be computed explicitly in terms of induction in relative Weyl groups. All of our calculations were done in the Chevie-system [21]. In addition, Harish-Chandra restriction ${ }^{*} R_{L}^{G}$ of projective characters also yields projective characters. This leads to the following indecomposability criterion:

$(\mathbf{H C r})$ Let $\chi$ be a projective character of $G$. If no proper subcharacter of $\chi$ has the property that its Harish-Chandra restriction to Levi subgroups $L$ decomposes nonnegatively on the PIMs of $L$, then $\chi$ is the character of a PIM.

One of our results is the subdivision into modular Harish-Chandra series of the Brauer characters in the block. A valuable criterion to determine this is given by [13, Thm. 4.2]:

(Csp) The group $G$ has a cuspidal unipotent Brauer character if and only if a Sylow $\ell$-subgroup of $G$ is not contained in any proper Levi subgroup of $G$.

Furthermore, the ordinary Gelfand-Graev character always provides the Steinberg PIM:

(St) There exists a PIM with unipotent part just the ordinary Steinberg character. It is non-cuspidal if and only if a Sylow $\ell$-subgroup of $G$ is contained in a proper Levi subgroup $L$. In this case it is a summand of the Harish-Chandra induction of the Steinberg PIM from $L$.

Other PIMs will appear as direct summands of generalized Gelfand-Graev representations (GGGRs). The results of Lusztig [20, §11], which have recently been extended to good characteristic by Taylor (see [23, Thm. 14.10]), give an approximation of some columns of the decomposition matrix:

(GGGR) Assume the underlying characteristic of $G$ is good. Given a unipotent character $\rho$, there exists a (projective) Gelfand-Graev representation $\Gamma$ such that $\rho$ occurs in $\Gamma$, and any other unipotent constituent in $\Gamma$ is either in the same family as $\rho$ or has a larger $a$-value.

Under suitable conditions on the family of unipotent characters containing $\rho$ one can even use [5, Thm. 6.5(ii)] to compute the multiplicities of the characters in the family in a GGGR. An instructive example is given in the proof of Theorem 3.6.

A further tool is given by a particular case of Dipper's result (see [6, 4.10] for the precise assumptions):

(End) The decomposition matrix of the Hecke algebra $\operatorname{End}_{G}\left(R_{T}^{G}\left(\mathbb{Z}_{\ell}\right)\right)$ embeds as a submatrix into the decomposition matrix of $G$.

Dipper's result holds more generally for a Hecke algebra associated with $R_{L}^{G}(\rho)$ where $\rho$ is a cuspidal unipotent character satisfying the following two conditions (see [11, §2.6]):

- the $\ell$-reduction of $\rho$ is an irreducible Brauer character $\varphi$,

- $N_{W}\left(W_{L}, \rho\right)=N_{W}\left(W_{L}, \varphi\right)$

where $W$ denotes the Weyl group of $G$. Note that when $L$ is classical, $\rho$ is the unique cuspidal unipotent character, so that $N_{W}\left(W_{L}, \rho\right)=N_{W}\left(W_{L}\right)$ and the second condition is automatically satisfied. The first condition is conjectured to hold whenever $\ell$ is good, as already mentioned in the introduction.

For dealing with PIMs which are not induced from a proper Levi subgroup, we will make use of suitably chosen Deligne-Lusztig characters as in [9] and [7, Sec. 6]: 
(DL) Let $\chi$ be a projective character of $G$. If $w \in W$ is minimal in the Bruhat order for the property that the unipotent part of $\chi$ occurs in the Deligne-Lusztig character $R_{w}$, then the sign of its multiplicity in $R_{w}$ is $(-1)^{\ell(w)}$.

We shall often use the following particular case:

(Cox) Let $\chi$ be the character of the projective cover of a cuspidal unipotent module, and $w \in W$ be a Coxeter element. Then the multiplicity of the unipotent part of $\chi$ in $(-1)^{\ell(w)} R_{w}$ is non-negative.

The previous two arguments will usually give upper bounds on decomposition numbers. Lower bounds can be obtained from $\ell$-reduction of non-unipotent characters, which are non-negative combinations of irreducible Brauer characters. This applies in particular to the Deligne-Lusztig induction of characters in general position:

(Red) Let $T_{w}$ be a torus of type $w \in W$. Assume that there exists an $\ell$-character of $T_{w}$ in general position. Then the $\ell$-reduction of $(-1)^{\ell(w)} R_{w}$ is a non-negative combination of irreducible Brauer characters.

Indeed, if $\theta$ is an $\ell$-character of $T_{w}$ in general position then $(-1)^{\ell(w)} R_{T_{w}}^{G}(\theta)$ is an irreducible character by [4] and it has the same $\ell$-reduction as $(-1)^{\ell(w)} R_{w}=(-1)^{\ell(w)} R_{T_{w}}^{G}\left(1_{T_{w}}\right)$.

We will also use variations of the following elementary observation:

(Sum) Let $\chi_{1}+\chi_{2}, \chi_{1}+\chi_{3}, \chi_{2}, \chi_{3}$ be characters of projectives modules, and assume that $\chi_{2}, \chi_{3}$ are indecomposable. Then $\chi_{1}$ is the character of a projective module.

Indeed, we have two direct sum decompositions of a projective module with character $\chi_{1}+\chi_{2}+\chi_{3}$, and the theorem of Krull-Schmidt allows to conclude. Sometimes, we will also make use of the following obvious fact:

(Deg) The degres of the irreducible Brauer characters in a block can be computed from the inverse of the decomposition matrix; they are all positive.

Our notation for modular Harish-Chandra series is as follows: characters in the principal series are labelled "ps", or sometimes "p" for short in large tables. If a Levi subgroup has a single cuspidal Brauer character, its Harish-Chandra series is labelled by the Dynkin type of that Levi subgroup. Else, it is labelled by the name of the corresponding ordinary unipotent character. For these, in turn, as customary we use the labelling in terms of ordinary Harish-Chandra series. Cuspidal Brauer characters are labelled by "c".

\section{Decomposition matrices for orthogonal groups of type $D_{n}$}

We first determine the decomposition matrices of orthogonal groups $\mathrm{SO}_{2 n}^{+}(q)$, with $n \leq 7$ and $q$ a prime power, for primes $2 \neq \ell \mid\left(q^{2}+1\right)$, except in the case that $\left(q^{2}+1\right)_{\ell}=5$. In the latter case, the decomposition matrices can be expected to be different from those in the general case, see Remarks 3.2 and 3.4 .

3.1. Decomposition matrices for $\mathrm{SO}_{8}^{+}(q)$. Let first $n=4$, so $G=\mathrm{SO}_{8}^{+}(q)$. For of the unipotent characters of $G$ lie in $\ell$-blocks of defect zero, the others lie in the principal block. Miyachi [22, Lemma 9] gives an approximation of the $\Phi_{4}$-modular decomposition matrix of the principal block of $\mathrm{SO}_{8}^{+}(q)$ when $q$ is odd, based on the triangularity of the decomposition matrix proved by Geck-Pfeiffer [16] using generalized Gelfand-Graev characters. Here we extend their results to all $q$ and determine the missing entry. 
Theorem 3.1. Let $\ell$ be a prime. The $\ell$-modular decomposition matrix for the principal block of $\mathrm{SO}_{8}^{+}(q), \ell \mid\left(q^{2}+1\right)$ with $\left(q^{2}+1\right)_{\ell}>5$, is as given in Table 1 .

TABLE $1 . \mathrm{SO}_{8}^{+}(q),\left(q^{2}+1\right)_{\ell}>5$

\begin{tabular}{|c|c|c|c|c|c|c|c|c|}
\hline .4 & 1 & 1 & & & & & & \\
\hline .31 & $q^{2} \Phi_{3} \Phi_{6}$ & 11 & & & & & & \\
\hline $2+$ & $q^{2} \Phi_{3} \Phi_{6}$ & 1. & 1 & & & & & \\
\hline $2-$ & $q^{2} \Phi_{3} \Phi_{6}$ & 1 & $\cdot$ & 1 & & & & \\
\hline 1.21 & $\frac{1}{2} q^{3} \Phi_{2}^{4} \Phi_{6}$ & 11 & 1 & 1 & 1 & & & \\
\hline$D_{4}$ & $\frac{1}{2} q^{3} \Phi_{1}^{4} \Phi_{3}$ & . & . & . & . 1 & & & \\
\hline $.21^{2}$ & $q^{6} \Phi_{3} \Phi_{6}$ & 1 & . & . & 1 . & 1 & & \\
\hline $1^{2}+$ & $q^{6} \Phi_{3} \Phi_{6}$ & . & 1 & . & 1 & . & 1 & \\
\hline $2-$ & $q^{6} \Phi_{3} \Phi_{6}$ & . & . & 1 & 1 . & . & . & 1 \\
\hline $.1^{4}$ & $q^{12}$ & . & . & . & 12 & 1 & 1 & $\begin{array}{ll}11 \\
\end{array}$ \\
\hline
\end{tabular}

Here, $D_{3}, A_{3}, A_{3}^{\prime}$ denote three non-conjugate Levi subgroups of type $A_{3}$.

Proof. We obtain all projectives in the table except for those in the 6th and 10th column by Harish-Chandra induction ( $\mathrm{HCi}$ ). The last column correspond to the Steinberg-PIM, which is cuspidal (St). The unipotent parts of the five principal series characters are precisely those of the Hecke algebra of type $D_{4}$ at a fourth root of unity by (End). Hence they are indecomposable for all $\ell$ dividing $q^{2}+1$. The printed PIMs in the series $A_{3}, A_{3}^{\prime}$ and $D_{3}$ are indecomposable by $(\mathrm{HCr})$.

Using the table of unipotent characters of $G$ in Chevie [21] one finds that the tensor product of the (projective) unipotent character $\rho_{.2^{2}}$ with the cuspidal unipotent character $\rho_{D_{4}}$ decomposes on the principal block as $(q-1) / 2 \rho_{D_{4}}+\left(q^{2}-1\right) / 4 \rho_{.1^{4}}$ for odd $q$, respectively as $q / 2 \rho_{D_{4}}+q^{2} / 4 \rho_{.1^{4}}$ when $q$ is even. This shows the existence of a PIM involving only the cuspidal unipotent character $\rho_{D_{4}}$ and an unknown multiple $a$ of the Steinberg character $\rho_{.1^{4}}$. In particular the decomposition matrix has unitriangular shape. Let $s_{1}, \ldots, s_{4}$ denote the simple reflections in the Weyl group of $G$. When $\left(q^{2}+1\right)_{\ell}>5$, there exists an $\ell-$ character in general position in the Sylow $\Phi_{4}$-torus $T_{w}$ for $w=\left(s_{1} s_{2} s_{3} s_{4}\right)^{2}$, forcing the relation $a \geq 2$ by (Red).

Finally, we use (Cox) to determine $a$ : the generalized 1-eigenspace of the Frobenius endomorphism on the Deligne-Lusztig character associated with a Coxeter element decomposes as

$$
\begin{aligned}
P_{s_{1} s_{2} s_{3} s_{4}} & =\rho_{.4}+\rho_{D_{4}}+\rho_{.1^{4}} \\
& =\Psi_{1}-\Psi_{2}-\Psi_{3}-\Psi_{4}+2 \Psi_{5}+\Psi_{6}-\Psi_{7}-\Psi_{8}-\Psi_{9}+(2-a) \Psi_{10},
\end{aligned}
$$

where $\Psi_{i}, i \neq 6$, denotes the unipotent part of the PIM corresponding to the $i$ th column in Table 1 and $\Psi_{6}:=\rho_{D_{4}}+a \rho_{.1^{4}}$, so that $a$ must be equal to 2 .

Remark 3.2. The 5-modular decomposition matrices of $\mathrm{SO}_{8}^{+}(2)$ and $\mathrm{SO}_{8}^{+}(3)$ are known; they differ from the one in Table 1 in that the entry " 2 " is replaced by " 1 ". Thus, Theorem 3.1 does not extend to the case $\left(q^{2}+1\right)_{\ell}=5$. 
3.2. Decomposition matrices for $\mathrm{SO}_{10}^{+}(q)$. We next consider the 10-dimensional orthogonal groups $G=\mathrm{SO}_{10}^{+}(q)$. Here, $G$ has four unipotent $\ell$-blocks when $\ell \mid\left(q^{2}+1\right)$, the principal block, one block with cyclic defect and two of defect zero, see e.g. [3].

Theorem 3.3. Let $\ell$ be a prime. The $\ell$-modular decomposition matrices for the unipotent blocks of $\mathrm{SO}_{10}^{+}(q)$ of positive defect for $\left(q^{2}+1\right)_{\ell}>5$ are as given in Tables 0 and 3 .

$$
\text { TABLE 2. } \mathrm{SO}_{10}^{+}(q),\left(q^{2}+1\right)_{\ell}>5
$$

\begin{tabular}{|c|c|c|c|c|c|c|c|c|c|c|c|c|c|c|}
\hline .5 & 1 & 1 & & & & & & & & & & & & \\
\hline 1.4 & $q \Phi_{5} \Phi_{6}$ & & 1 & & & & & & & & & & & \\
\hline 2.3 & $q^{2} \Phi_{5} \Phi_{8}$ & 1 & 1 & 1 & & & & & & & & & & \\
\hline .32 & $\frac{1}{2} q^{3} \Phi_{5} \Phi_{6} \Phi_{8}$ & 1 & . & . & 1 & & & & & & & & & \\
\hline 1.31 & $\frac{1}{2} q^{3} \Phi_{3} \Phi_{5} \Phi_{8}$ & . & 1 & 1 & & 1 & & & & & & & & \\
\hline$D_{4}: 2$ & $\frac{1}{2} q^{3} \Phi_{1}^{4} \Phi_{3} \Phi_{5}$ & . & . & . & & . & 1 & & & & & & & \\
\hline $1.2^{2}$ & $q^{5} \Phi_{5} \Phi_{6} \Phi_{8}$ & 1 & . & 1 & 1 & . & . & 1 & & & & & & \\
\hline $.31^{2}$ & $q^{6} \Phi_{3} \Phi_{6} \Phi_{8}$ & . & . & . & . & 1 & . & . & 1 & & & & & \\
\hline $.2^{2} 1$ & $\frac{1}{2} q^{7} \Phi_{5} \Phi_{6} \Phi_{8}$ & . & . & . & 1 & . & . & 1 & . & 1 & & & & \\
\hline $1.21^{2}$ & $\frac{1}{2} q^{7} \Phi_{3} \Phi_{5} \Phi_{8}$ & . & . & 1 & & 1 & . & . & 1 & . & 1 & & & \\
\hline$D_{4}: 1^{2}$ & $\frac{1}{2} q^{7} \Phi_{1}^{4} \Phi_{3} \Phi_{5}$ & . & . & . & & . & . & . & . & . & . & 1 & & \\
\hline $1^{2} .1^{3}$ & $q^{10} \Phi_{5} \Phi_{8}$ & . & . & 1 & & . & . & 1 & . & . & 1 & & 1 & \\
\hline $1.1^{4}$ & $q^{13} \Phi_{5} \Phi_{6}$ & . & . & . & & . & 2 & . & 1 & . & 1 & . & 1 & 1 \\
\hline $.1^{5}$ & $q^{20}$ & . & . & . & . & . & . & 1 & . & 1 & . & 2 & 1 & . 1 \\
\hline
\end{tabular}

TABLE $3 . \mathrm{SO}_{10}^{+}(q)$, block of defect $1,2 \neq \ell \mid\left(q^{2}+1\right)$

$$
.41-2.21-1^{2} .21-21^{3} \frac{-}{D_{3}} \bigcirc
$$

Proof. The Brauer tree for the block with cyclic defect is easily determined (see also [10]), so it remains to consider the principal block. Let us denote by $\Psi_{1}, \ldots, \Psi_{14}$ the linear combinations of unipotent characters given by the columns in Table 2. We shall show that these are the unipotent parts of projective indecomposable characters of $G$.

Using ( $\mathrm{HCi}$ ) gives $\Psi_{i}$ for $i \in\{2,3,4,5,8,10,12\}$. The decomposition matrix of the Hecke algebra of type $D_{5}$ at a fourth root of unity gives by (End) the seven principal series PIMs $\Psi_{1}, \ldots, \Psi_{5}, \Psi_{7}$ and $\Psi_{10}$ for all primes $\ell$ with $\left(q^{2}+1\right)_{\ell}>5$. Furthermore, (HCi) yields $\tilde{\Psi}_{9}:=\Psi_{9}+\Psi_{8}$ and $\tilde{\Psi}_{10}:=\Psi_{9}+\Psi_{10}$. An application of (Sum) yields $\Psi_{9}$.

The centralizer of a Sylow $\Phi_{4}$-torus of $G$ is contained in a Levi subgroup $L$ of type $D_{4}$, so [13, Thm. 4.2] shows that the Harish-Chandra induction $\tilde{\Psi}_{13}=\Psi_{13}+\Psi_{14}$ of the Steinberg PIM from $L$ has two summands, namely $\Psi_{13}$ and $\Psi_{14}$. 
(HCi) also yields a projective character with unipotent part $\tilde{\Psi}_{6}=\Psi_{6}+\Psi_{11}$. The Hecke algebra for the ordinary cuspidal character of a Levi subgroup $L \leq G$ of type $D_{4}$ has type $A_{1}$ with parameter $q^{4}$, hence is semisimple modulo $\ell$, so the Harish-Chandra induction of the corresponding PIM from $L$ has two summands in that Harish-Chandra series. Decomposition of the Harish-Chandra restriction to the proper Levi subgroups shows that these summands must have the form $\Psi_{6}-(2-a)\left(\Psi_{13}-\Psi_{14}\right)$ and $\Psi_{11}+(2-a)\left(\Psi_{13}-\Psi_{14}\right)$, with one undetermined parameter $a \in\{0,1,2\}$.

Finally, using $(\mathrm{HCr})$ we check that, independently from the value of $a$, all $\Psi_{i}$ are indecomposable. Indeed, no proper subsums restrict to a non-negative combination of PIMs in all Levi subgroups. We shall prove that $a=2$ in Theorem 3.6 below.

Remark 3.4. The 5-modular decomposition matrix of $\mathrm{SO}_{10}^{+}(2)$ is known; it differs from the one in Table 2 in that the two entries "2" are replaced by " 1 "s. Thus, Theorem 3.3 does not extend to the case where $\left(q^{2}+1\right)_{\ell}=5$.

3.3. Decomposition matrices for $\mathrm{SO}_{12}^{+}(q)$. Now let $G=\mathrm{SO}_{12}^{+}(q)$. This group has four unipotent $\Phi_{4}$-blocks of defect zero and three blocks of defect $\Phi_{4}^{2}$. We label these blocks by the symbol for their 4-Harish-Chandra source in a 4-split Levi subgroup of type $\mathrm{SO}_{4}^{+}(q)$.

Theorem 3.5. Let $\ell$ be a prime. The $\ell$-modular decomposition matrices for the unipotent blocks of $\mathrm{SO}_{12}^{+}(q)$ of positive defect, for $\left(q^{2}+1\right)_{\ell}>5$, are as given in Tables 46 .

TABLE $4 . \mathrm{SO}_{12}^{+}(q)$, block $\left(\begin{array}{l}2 \\ 0\end{array}\right)$, and $\mathrm{SO}_{16}^{+}(q)$, block $\left(\begin{array}{l}3 \\ 1\end{array}\right),\left(q^{2}+1\right)_{\ell}>5$

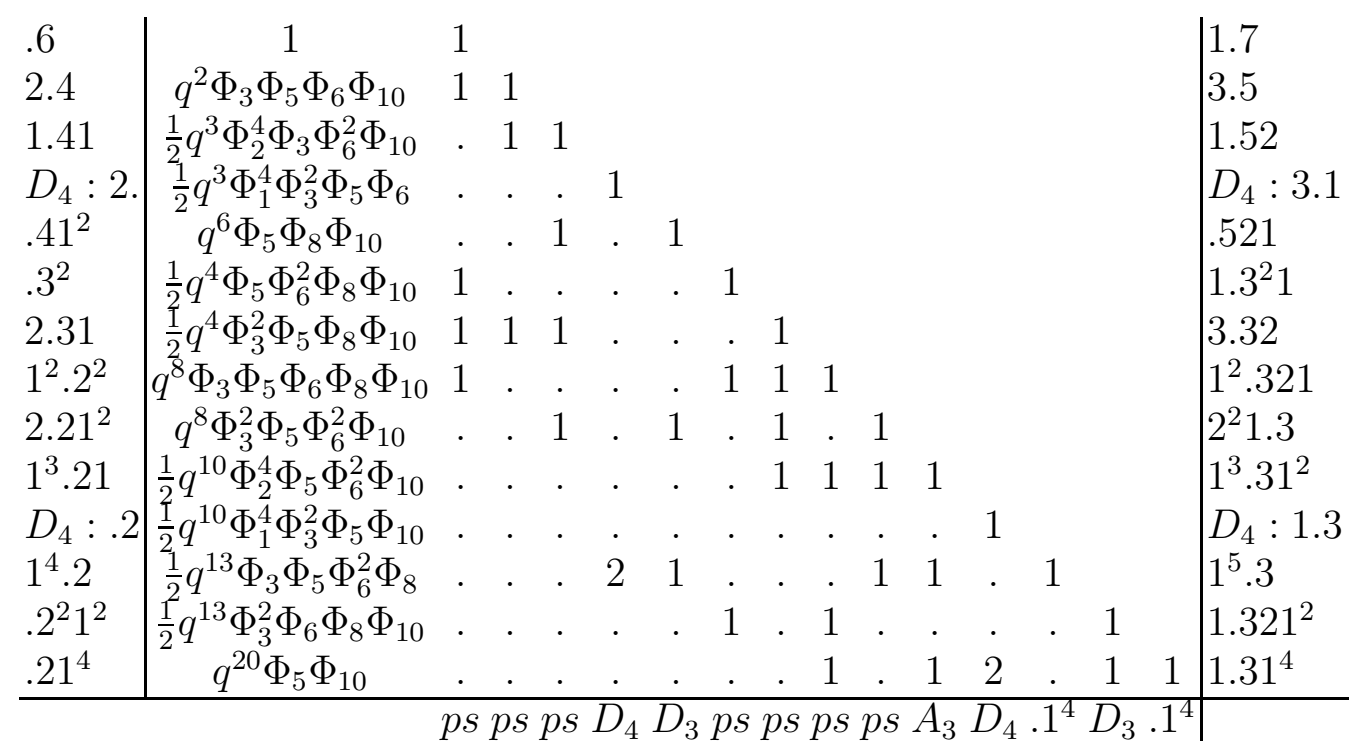

Proof. All projectives listed in the tables are obtained by (HCi) from the Levi subgroups of types $D_{5}$ and $A_{5}$, except that instead of $\Psi_{4}$ and $\Psi_{11}$ in the first block we obtain $\Psi_{4}-(2-a)\left(\Psi_{12}-\Psi_{14}\right)$ and $\Psi_{11}+(2-a)\left(\Psi_{12}-\Psi_{14}\right)$, and instead of $\Psi_{5}$ and $\Psi_{12}$ in the third block we obtain $\Psi_{5}-(2-a)\left(\Psi_{11}-\Psi_{14}\right)$ and $\Psi_{12}+(2-a)\left(\Psi_{11}-\Psi_{14}\right)$, with the parameter $a \in\{0,1,2\}$ as in the proof of Theorem 3.3. It is straightforward to check by 
TABLE 5. $\mathrm{SO}_{12}^{+}(q)$, block $\left(\begin{array}{l}1 \\ 1\end{array}\right),\left(q^{2}+1\right)_{\ell}>5$

\begin{tabular}{|c|c|c|c|c|c|c|c|c|c|}
\hline 1.5 & $q \Phi_{3} \Phi_{6} \Phi_{8}$ & 1 & & & & & & & \\
\hline $3+$ & $q^{3} \Phi_{5} \Phi_{8} \Phi_{10}$ & 11 & & & & & & & \\
\hline $3-$ & $q^{3} \Phi_{5} \Phi_{8} \Phi_{10}$ & 1. & 1 & & & & & & \\
\hline 1.32 & $q^{5} \Phi_{3} \Phi_{5} \Phi_{6} \Phi_{8} \Phi_{10}$ & 11 & 1 & 1 & & & & & \\
\hline .321 & $\frac{1}{2} q^{7} \Phi_{2}^{4} \Phi_{6}^{2} \Phi_{8} \Phi_{10}$ & . . & . & 1 & 1 & & & & \\
\hline$D_{4}: 1.1$ & $\frac{1}{2} q^{7} \Phi_{1}^{4} \Phi_{3}^{2} \Phi_{5} \Phi_{8}$ & . & . & . & . & 1 & & & \\
\hline $1.2^{2} 1$ & $q^{9} \Phi_{3} \Phi_{5} \Phi_{6} \Phi_{8} \Phi_{10}$ & 1 & 1 & 1 & 1 & . & 1 & & \\
\hline $1^{3}+$ & $q^{15} \Phi_{5} \Phi_{8} \Phi_{10}$ & 1 & . & . & . & . & 1 & 1 & \\
\hline $1^{3}-$ & $q^{15} \Phi_{5} \Phi_{8} \Phi_{10}$ & . & 1 & . & . & . & 1 & . & 1 \\
\hline $1.1^{5}$ & $q^{21} \Phi_{3} \Phi_{6} \Phi_{8}$ & . & & . & 1 & 2 & 1 & 1 & 1 \\
\hline
\end{tabular}

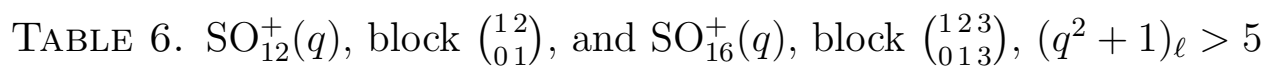

\begin{tabular}{|c|c|c|c|c|c|c|c|c|c|c|c|c|c|c|}
\hline .51 & $q^{2} \Phi_{5} \Phi_{10}$ & 1 & & & & & & & & & & & & $1.51^{2}$ \\
\hline .42 & $\frac{1}{2} q^{3} \Phi_{3}^{2} \Phi_{6} \Phi_{8} \Phi_{10}$ & 11 & 1 & & & & & & & & & & & 1.421 \\
\hline $1^{2} .4$ & $\frac{1}{2} q^{3} \Phi_{3} \Phi_{5} \Phi_{6}^{2} \Phi_{8}$ & - & 1 & & & & & & & & & & & $1^{3} .5$ \\
\hline 21.3 & $\frac{1}{2} q^{4} \Phi_{2}^{4} \Phi_{5} \Phi_{6}^{2} \Phi_{10}$ & 1 & 1 & 1 & & & & & & & & & & $3.31^{2}$ \\
\hline$D_{4}: 1^{2}$. & $\frac{1}{2} q^{4} \Phi_{1}^{4} \Phi_{3}^{2} \Phi_{5} \Phi_{10}$ & . & . & . & 1 & & & & & & & & & $D_{4}: 1^{3} .1$ \\
\hline $2.2^{2}$ & $q^{6} \Phi_{3} \Phi_{5} \Phi_{6} \Phi_{8} \Phi_{10}$ & 1 & . & 1 & . & 1 & & & & & & & & 2.321 \\
\hline $1^{2} .31$ & $q^{6} \Phi_{3}^{2} \Phi_{5} \Phi_{6}^{2} \Phi_{10}$ & . & 1 & 1 & . & & 1 & & & & & & & $1^{3} .32$ \\
\hline $.31^{3}$ & $q^{12} \Phi_{5} \Phi_{8} \Phi_{10}$ & . & . & . & . & . & 1 & 1 & & & & & & $.321^{3}$ \\
\hline $.2^{3}$ & $\frac{1}{2} q^{10} \Phi_{5} \Phi_{6}^{2} \Phi_{8} \Phi_{10}$ & . & 1 & . & . & 1 & . & . & 1 & & & & & $1.32^{2}$ \\
\hline $1^{2} .21^{2}$ & $\frac{1}{2} q^{10} \Phi_{3}^{2} \Phi_{5} \Phi_{8} \Phi_{10}$ & . & . & 1 & . & 1 & 1 & . & . & 1 & & & & $1^{3} \cdot 2^{2} 1$ \\
\hline $1.21^{3}$ & $\frac{1}{2} q^{13} \Phi_{2}^{4} \Phi_{3} \Phi_{6}^{2} \Phi_{10}$ & . & . & . & 2 & . & 1 & 1 & & 1 & 1 & & & $1.2^{2} 1^{3}$ \\
\hline$D_{4}: .1^{2}$ & $\frac{1}{2} q^{13} \Phi_{1}^{4} \Phi_{3}^{2} \Phi_{5} \Phi_{6}$ & . & . & . & . & . & . & . & . & . & . & 1 & & $D_{4}: 1.1^{3}$ \\
\hline $1^{2} .1^{4}$ & $q^{16} \Phi_{3} \Phi_{5} \Phi_{6} \Phi_{10}$ & . & . & . & 2 & 1 & . & . & . & 1 & 1 & & 1 & $1^{3} \cdot 1^{5}$ \\
\hline $.1^{6}$ & $q^{30}$ & . & . & . & . & 1 & . & . & 1 & . & . & 2 & 11 & $1.1^{7}$ \\
\hline
\end{tabular}

(HCr) that all these projective characters are indecomposable. We shall prove that $a=2$ in Theorem 3.6 below.

3.4. Decomposition matrices for $\mathrm{SO}_{14}^{+}(q)$. We now consider the three blocks of positive $\Phi_{4}$-defect for the groups $G=\mathrm{SO}_{14}^{+}(q)$. The non-principal block is again labelled by the symbol for its 4-Harish-Chandra source in a 4-split Levi subgroup of type $\mathrm{SO}_{6}^{+}(q)$.

Theorem 3.6. Let $G=\mathrm{SO}_{14}^{+}(q)$ and $\ell$ a prime with $\left(q^{2}+1\right)_{\ell}>5$.

(a) If $q$ is odd then the decomposition matrix for the principal $\ell$-block of $G$ is as given in Tables 7 and 8 . 
(b) The decomposition matrix for the non-principal unipotent $\ell$-block $G$ of positive defect is as given in Table 9 .

Proof. Let us first consider the block with defect $\Phi_{4}^{2}$. We argue how to construct projectives $\Psi_{1}, \ldots, \Psi_{14}$ with unipotent part equal to the columns in Table 9. (HCi) and (End) give all $\Psi_{i}$, except that instead of $\Psi_{4}$ and $\Psi_{13}$ we find $\Psi_{4}-(2-a)\left(\Psi_{10}-\Psi_{14}\right)$ and $\Psi_{13}+(2-a)\left(\Psi_{10}-\Psi_{14}\right)$, with $a \in\{1,2\}$ as in the proof of Theorem 3.5. Again, it is easily seen by $(\mathrm{HCr})$ that all these characters are indecomposable. Now the tenth Brauer character has positive degree only if $a \geq 2$, which shows by (Deg) that $a=2$ in this table and also in the decomposition matrices for $\mathrm{SO}_{10}^{+}(q)$ and $\mathrm{SO}_{12}^{+}(q)$, thus completing the proofs of Theorems 3.3 and 3.5. Note that up to this point we did not use any assumption on $q$.

We now turn to the principal block. Here, (HCi) yields $\Psi_{i}$ except for $i \in\{2,14,16,19$, $24,25,26,34,35,36,38,40\}$. Using (Sum) we get other projective characters: $\Psi_{2}+\Psi_{3}$ and $\Psi_{2}+\Psi_{4}+\Psi_{10}$ give $\Psi_{2}, \Psi_{14}+\Psi_{17}$ and $\Psi_{14}+\Psi_{29}$ give $\Psi_{14}, \Psi_{6}+\Psi_{19}$ and $\Psi_{19}+\Psi_{30}$ give $\Psi_{19}$, $\Psi_{12}+\Psi_{16}$ and $\Psi_{16}+\Psi_{19}$ give $\Psi_{16}, \Psi_{9}+\Psi_{20}+\Psi_{24}$ and $\Psi_{24}+\Psi_{27}$ give $\Psi_{24}, \Psi_{31}+\Psi_{35}$ and $\Psi_{33}+\Psi_{35}$ give $\Psi_{35}, \Psi_{28}+\Psi_{34}$ and $\Psi_{34}+\Psi_{35}$ give $\Psi_{34}$. Furthermore we find $\Psi_{26}$ with $c=1$. The PIM $\Psi_{40}$ is cuspidal by ( $\mathrm{St}$ ). ( $\mathrm{HCr}$ ) shows that all of the projectives obtained so far, with the possible exception of $\Psi_{26}$, which might contain $\Psi_{27}$ once, are indecomposable. We have thus obtained all but three columns of the decomposition matrix. Since we have accounted for all proper Harish-Chandra series, the remaining three Brauer characters must be cuspidal.

To establish the unitriangularity we look at suitably chosen generalized Gelfand-Graev representations and use (GGGR). Note that we have to assume that $q$ is odd in order to construct these representations and use the results in [23]. Let us first consider the family $\mathcal{F}=\left\{\rho_{1^{5} .2}, \rho_{1.21^{4}}, \rho_{.2^{2} 1^{3}}, \rho_{D_{4}: 1^{3}}\right\}$ of unipotent characters. The special character of this family is $\rho_{1.21^{4}}$; via the Springer correspondence, it corresponds to a special unipotent class, and we denote by $\mathcal{O}$ its dual. By [23, Thm. 14.10], the character of any GGGR attached to $\mathcal{O}$ involves characters lying in $\mathcal{F}$ or in a family with a strictly larger $a$ value than that of $\mathcal{F}$. In particular, the only characters in the block that can occur are $\rho_{1^{5} .2}, \rho_{1.21^{4}}, \rho_{D_{4}: .1^{3}}, \rho_{1^{3} .1^{4}}, \rho_{1^{2} .1^{5}}, \rho_{1.1^{6}}$ and $\rho_{.1^{7}}$, which gives an approximation of $\Psi_{36}$. Furthermore, $u \in \mathcal{O}^{F}$ satisfies the following two conditions:

- the small finite group attached to the family as in [19, Chap. 4] and the component group $A_{G}(u):=\left(C_{\mathbf{G}}(u) / C_{\mathbf{G}}(u)^{\circ}\right)^{F}$ are isomorphic (to $\mathbb{Z} / 2 \mathbb{Z}$ ),

- at most one of the local systems on $(u)$ is not in the principal block,

in which case one can apply [5, Thm. 6.5(ii)] to compute the projection of the GGGR $\Gamma_{u}$ into the span of $\mathcal{F}$. Recall that the characters in a family are parametrized by pairs $(g, \psi)$ where $g$ runs over a set of representatives of conjugacy classes of the small finite group attached to the family, say $\mathcal{G}$ and $\psi \in \operatorname{Irr}\left(C_{\mathcal{G}}(g)\right)$. For $g \in \mathcal{G}$, the Mellin transform of the pair $(g, 1)$ is given by

$$
\mu_{(g, 1)}=\sum_{\psi \in \operatorname{Irr}\left(C_{\mathcal{G}}(g)\right)} \psi(g) \rho_{(g, \psi)} .
$$


TABLE $7 . \mathrm{SO}_{14}^{+}(q), q$ odd, principal block, $\left(q^{2}+1\right)_{\ell}>5$

\begin{tabular}{|c|c|c|c|c|c|c|c|c|c|c|c|c|c|c|c|c|c|c|c|c|}
\hline 7 & 1 & & & & & & & & & & & & & & & & & & & \\
\hline 1.6 & 1 & 1 & & & & & & & & & & & & & & & & & & \\
\hline 2.5 & 1 & . & 1 & & & & & & & & & & & & & & & & & \\
\hline 1.51 & & . & 1 & 1 & & & & & & & & & & & & & & & & \\
\hline $1^{2} .5$ & . & 1 & . & . & 1 & & & & & & & & & & & & & & & \\
\hline$D_{4}: 3$. & & . & & . & & 1 & & & & & & & & & & & & & & \\
\hline 3.4 & 1 & 1 & 1 & . & & . & 1 & & & & & & & & & & & & & \\
\hline 3.31 & 1 & 1 & 1 & 1 & 1 & . & 1 & 1 & & & & & & & & & & & & \\
\hline 1.42 & 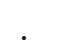 & . & 1 & 1 & & . & 1 & . & 1 & & & & & & & & & & & \\
\hline $1.3^{2}$ & 1 & 1 & . & . & & . & 1 & . & . & 1 & & & & & & & & & & \\
\hline 2.32 & 1 & . & 1 & 1 & . & . & 1 & 1 & 1 & . & 1 & & & & & & & & & \\
\hline$D_{4}: 1^{3}$. & . & . & . & . & . & . & . & . & . & . & . & 1 & & & & & & & & \\
\hline $.51^{2}$ & . & . & . & 1 & . & . & . & . & . & . & . & . & 1 & & & & & & & \\
\hline .421 & . & . & . & 1 & . & . & . & . & 1 & . & . & . & 1 & 1 & & & & & & \\
\hline $1^{3} .4$ & . & . & . & . & 1 & . & . & . & . & . & . & . & . & . & 1 & & & & & \\
\hline$D_{4}: 2.1$ & . & . & . & . & . & 1 & . & . & . & . & . & . & . & . & . & 1 & & & & \\
\hline $.3^{2} 1$ & 1 & . & . & . & . & . & . & . & . & 1 & . & . & . & . & . & . & 1 & & & \\
\hline $21^{2} .3$ & . & . & . & 1 & 1 & . & . & 1 & . & . & . & . & 1 & . & 1 & . & . & 1 & & \\
\hline$D_{4}: 1^{2} .1$ & & . & . & . & & . & . & . & . & . & . & 1 & . & . & . & . & . & & 1 & \\
\hline $1^{2} .32$ & 1 & 1 & . & . & 1 & . & 1 & 1 & . & 1 & . & . & . & . & . & . & . & . & . & 1 \\
\hline $1^{3} .31$ & . & . & . & . & 1 & . & . & 1 & . & . & . & . & . & . & 1 & . & . & 1 & . & 1 \\
\hline $.32^{2}$ & . & . & . & . & . & . & . & . & 1 & . & 1 & . & . & 1 & . & . & . & . & . & . \\
\hline$D_{4}: 1.2$ & . & . & . & . & . & . & . & . & . & . & . & . & . & . & . & . & . & . & 1 & . \\
\hline $2.2^{2} 1$ & . & . & . & 1 & & . & 1 & 1 & 1 & . & 1 & . & 1 & 1 & . & . & . & 1 & . & . \\
\hline$D_{4}: .3$ & . & . & . & . & . & . & . & . & . & . & . & . & . & . & . & . & . & . & . & . \\
\hline $1.2^{3}$ & . & . & . & . & . & . & 1 & . & 1 & . & 1 & . & . & 1 & . & . & . & . & . & . \\
\hline $1^{2} \cdot 2^{2} 1$ & 1 & . & . & . & & . & 1 & 1 & . & 1 & 1 & . & . & . & . & . & 1 & . & . & 1 \\
\hline $1^{4} .3$ & . & . & . & . & . & 2 & . & . & . & . & . & . & 1 & . & 1 & . & . & 1 & . & . \\
\hline $.321^{2}$ & . & . & . & . & . & . & . & . & . & 1 & . & . & . & . & . & . & 1 & . & . & 1 \\
\hline$D_{4}: 1.1^{2}$ & . & . & . & . & & . & $\cdot$ & . & . & . & . & & . & . & . & 1 & & . & . & $\cdot$ \\
\hline $1.2^{2} 1^{2}$ & . & . & . & . & & . & 1 & . & . & 1 & . & 2 & . & . & . & . & 1 & . & . & 1 \\
\hline $1^{3} .21^{2}$ & . & . & . & . & . & . & 1 & 1 & . & . & 1 & . & . & . & . & . & . & 1 & . & 1 \\
\hline $.31^{4}$ & . & . & . & . & • & . & . & . & . & . & . & . & . & . & . & . & . & . & . & 1 \\
\hline $1.21^{4}$ & . & . & . & . & & . & . & . & . & . & . & 2 & . & . & . & . & 1 & . & 2 & 1 \\
\hline $1^{5} .2$ & . & . & . & . & & 2 & & . & . & . & . & . & 1 & 1 & . & 2 & & 1 & . & . \\
\hline$D_{4}: .1^{3}$ & . & . & . & · & - & $\cdot$ & & · & . & - & $\cdot$ & & . & $\cdot$ & $\cdot$ & $\cdot$ & . & $\cdot$ & $\cdot$ & $\cdot$ \\
\hline $1^{3} .1^{4}$ & . & . & . & . & $\cdot$ & . & 1 & . & . & · & 1 & 2 & . & . & . & . & . & . & . & . \\
\hline $1^{2} .1^{5}$ & . & . & . & . & . & . & . & . & . & . & 1 & 2 & . & . & . & . & 1 & . & 2 & . \\
\hline $1.1^{6}$ & . & . & & . & & . & & . & & & 1 & & . & 1 & . & 2 & & . & . & . \\
\hline $.1^{7}$ & & . & & . & & & & . & & . & 1 & & . & . & . & . & & . & & \\
\hline
\end{tabular}


TABLE 8. $\mathrm{SO}_{14}^{+}(q)$, principal block, cntd.

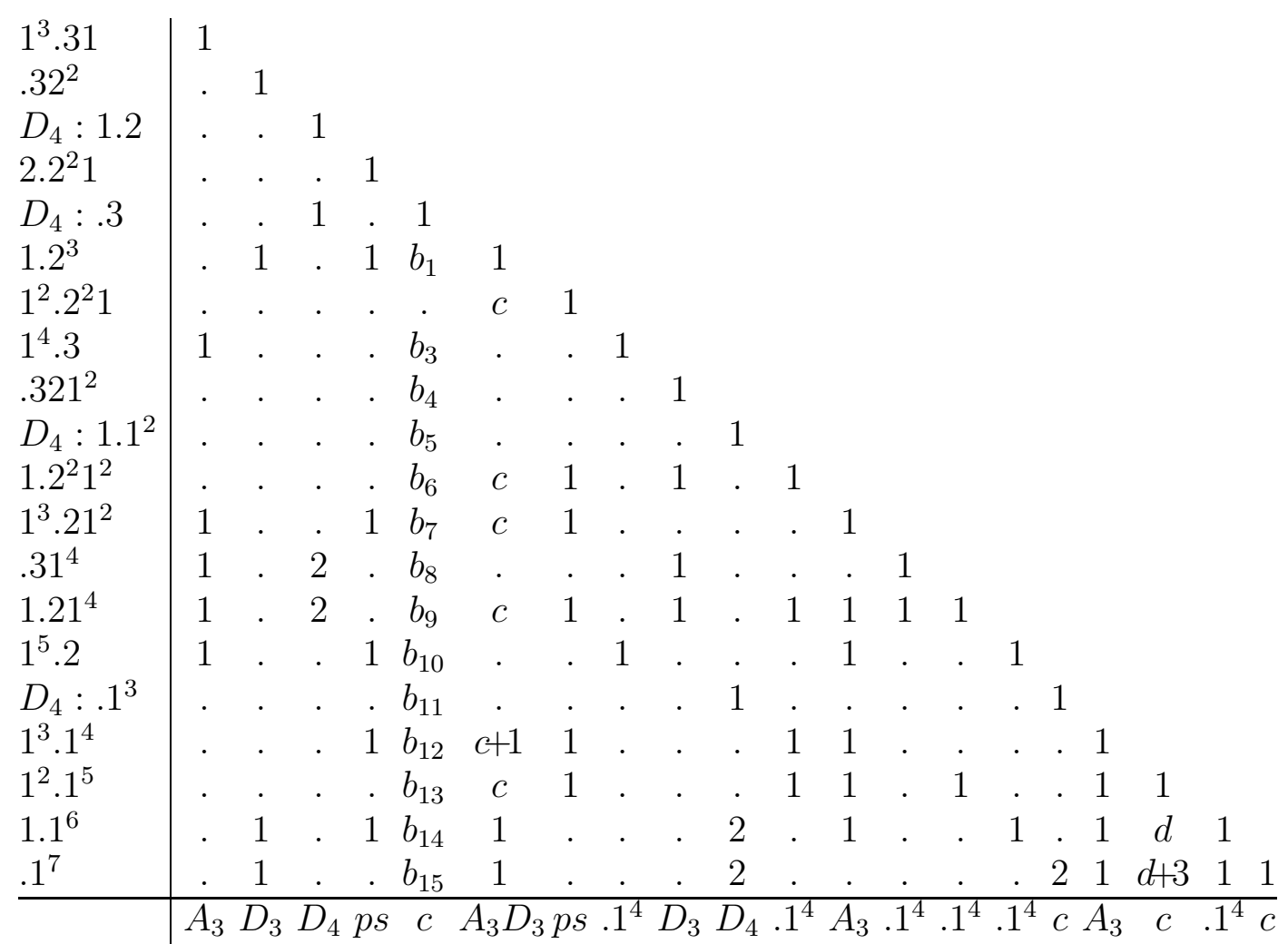

Here, $b_{1}, c, d \in\{0,1\}$. Moreover, if $p>5$ then $b_{3}=b_{4}=b_{5}=b_{7}=0$ and $b_{6} \in\{0,1\}$.

In particular, the small finite group for the dual family of $\mathcal{F}$ is $\mathbb{Z} / 2 \mathbb{Z}$ and the Mellin transforms of $(1,1)$ and $(-1,1)$ are

$$
\begin{aligned}
\mu_{(1,1)}=\rho_{(1,1)}+\rho_{(1, \varepsilon)} & =\rho_{1.51}+\rho_{1^{2} .5}, \\
\mu_{(-1,1)}=\rho_{(-1,1)}-\rho_{(-1, \varepsilon)} & =\rho_{.52}-\rho_{D_{4}: 3 .},
\end{aligned}
$$

where $\varepsilon$ denotes the non-trivial character of $\mathbb{Z} / 2 \mathbb{Z}$. By [5, Thm. 6.5(ii)], the projections to $\mathcal{F}$ of the two GGGRs attached to $\mathcal{O}$ are given by the Alvis-Curtis duals of these characters, that is by

$$
\rho_{1.21^{4}}+\rho_{1^{5} .2} \text { and } \rho_{.2^{2} 1^{3}}+\rho_{D_{4}: 1^{3}} \text {. }
$$

Taking the second GGGR and cutting by the block, we obtain a projective character whose unipotent part is given by $\rho_{D_{4}: 1^{3}}+c_{1} \rho_{1^{3} .1^{4}}+c_{2} \rho_{1^{2} .1^{5}}+c_{3} \rho_{1.1^{6}}+c_{4} \rho_{.1^{7}}$. Note that $c_{1}$ is actually zero by [23, Thm. 14.10] since $\rho_{1^{3} .1^{4}}$ and $\rho_{D_{4}: 1^{3}}$ have the same $a$-value but lie in different families.

For $\Psi_{25}$ and $\Psi_{38}$ we consider the GGGRs associated with the families $\left\{\rho_{1^{2} .2^{2} 1}, \rho_{1^{3} .2^{2}}, \rho_{1.2^{3}}\right.$, $\left.\rho_{D_{4}: 3}\right\}$ and $\left\{\rho_{1^{2} .1^{5}}\right\}$, from which we deduce that the decomposition matrix is unitriangular. Moreover, if we denote by $\left(b_{i}\right)_{i=1, \ldots, 15}$ (resp. $\left.c_{5}, c_{6}\right)$ the unknown entries in the 25 th (resp. 38th) column then [5, Thm. 6.5] yields $b_{2}=0$.

The unipotent part of the Gelfand-Graev representation of $\mathrm{SO}_{16}^{+}(q)$ associated with the family $\left\{\rho_{1^{2} .1^{6}}\right\}$, cut by the principal block, is of the form $\rho_{1^{2} .1^{6}}+\alpha \rho_{2.1^{6}}+\beta \rho_{.1^{8}}$ for 
TABLE $9 . \mathrm{SO}_{14}^{+}(q)$, block $\left(\begin{array}{l}13 \\ 01\end{array}\right),\left(q^{2}+1\right)_{\ell}>5$

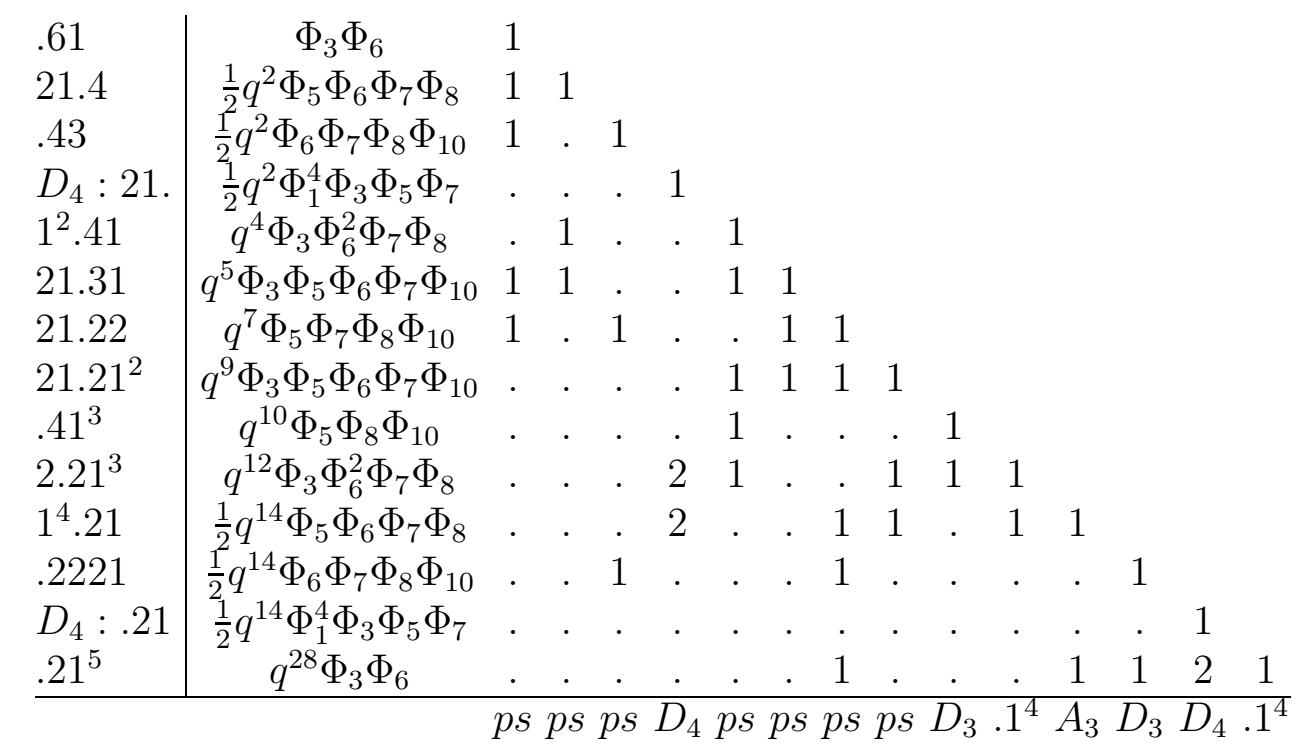

Here, all character degrees have been divided by $q^{2} \Phi_{4} \Phi_{12}$.

suitable $\alpha, \beta \geq 0$. The Harish-Chandra restriction of this character to $\mathrm{SO}_{14}^{+}(q)$, cut by the principal block, equals $\rho_{1^{2} .1^{5}}+\rho_{1.1^{6}}+(\alpha+\beta) \rho_{.1^{7}}$ and thus forces $c_{5} \leq 1$.

If moreover $p>5$ we may also consider a GGGR associated to the family $\left\{\phi_{700,42}\right.$, $\left.\phi_{400,43}, \phi_{300,44}, D_{4}: \phi_{1,12}^{\prime \prime}\right\}$ of $E_{8}$, whose projection on the family is $\phi_{400,43}+D_{4}: \phi_{1,12}^{\prime \prime}$. By [23, Thm. 14.10], the only other unipotent characters lying in the principal $\ell$-block which can occur as constituents are $\phi_{1,120}, \phi_{35,74}, \phi_{50,56}, \phi_{210,52}, \phi_{567,46}, \phi_{112,63}$ and $D_{4}: \phi_{1,24}$. As above, the Harish-Chandra reduction of the GGGR gives upper bounds for some of the $b_{i}$ 's, namely $b_{3}=b_{4}=b_{5}=b_{7}=0$ and $b_{6} \leq 1$

We now use (DL) to obtain relations on the other decomposition numbers. Let $w$ be a Coxeter element. For $v<w$, one checks easily that the characters $\Psi_{38}, \Psi_{39}$ and $\Psi_{40}$ do not occur in $R_{v}$. Therefore the computation of $R_{w}$ yields by (DL) three inequalities which are $-c_{2} \geq 0, c_{2} c_{5}-c_{3} \geq 0$ and $2+c_{3}-c_{4}+c_{2}\left(c_{6}-c_{5}\right) \geq 0$. This forces $c_{2}=c_{3}=0$ and $c_{4} \leq 2$. We use (Red) to prove that $c_{4}=2$. More precisely, we consider the $\ell$-reduction of a non-unipotent character which is obtained by inducing an $\ell$-character in general position of a $\Phi_{4}$-torus (of order $\left.(q+1)\left(q^{2}+1\right)^{3}\right)$. Such a character exists whenever $\left(q^{2}+1\right)_{\ell}>12$, which automatically holds if $\left(q^{2}+1\right)_{\ell}>5$. This yields the relations $c_{4} \geq 2$ and $c_{6} \geq c_{5}+3$, so that $c_{4}=2$. In particular, none of $\Psi_{38}, \Psi_{39}$ and $\Psi_{40}$ occur in $R_{w}$.

Finally, we use (DL) with $w^{\prime}=s_{1} s_{3} s_{1} s_{2} s_{3} s_{4} s_{5} s_{6} s_{7}$, where $s_{1}, \ldots, s_{7}$ are the simple reflections ordered as in Chevie (so the end nodes are 1,2 and 7), and we find $c_{5}+3 \geq c_{6}$, so that $c_{6}=c_{5}+3$. Note that one relation on the $b_{i}$ 's can also be obtained. The resulting decomposition matrix is given in Table 8, where $c_{5} \in\{0,1\}$ is simply denoted by $d$.

Remark 3.7. Under some assumptions on the special unipotent class $\mathcal{O}$, Kawanaka conjectured in [17] that one can decompose any GGGR associated with $\mathcal{O}$ into a sum of 
projective characters, each of which contains only one unipotent character of the family. If Kawanaka's conjecture holds for the family $\left\{1^{2} .2^{2} 1,1^{3} .2^{2}, 1.2^{3}, D_{4}: .3\right\}$ of $\mathrm{SO}_{14}(q)$, then $b_{1}$ and $d$ must be equal to zero. More generally, as suggested by Geck, Kawanaka's characters should force block unitriangularity of the decomposition matrix whenever $p$ is good.

Our method is not sufficient to determine all the decomposition numbers of $\mathrm{SO}_{14}^{+}(q)$. However, we can use [8, Conj. 1.2] to determine small upper bounds for the missing entries. Following [8], we denote by $Q_{w}$ the virtual character afforded by the Alvis-Curtis dual of the intersection cohomology of the Deligne-Lusztig variety corresponding to $w$. In addition, if $\lambda \in \overline{\mathbb{F}}_{\ell}$ we consider the virtual character $Q_{w}[\lambda]$ afforded by the generalized $\lambda$-eigenspace of the Frobenius on the intersection cohomology. Up to a sign, $Q_{w}[\lambda]$ is a proper character and Conjecture 1.2 in [8] predicts that it is actually the unipotent part of a projective character. The multiplicities of the various PIMs in $Q_{w}[\lambda]$ depend on the decomposition numbers (including the missing entries), forcing some linear combinations of decomposition numbers to be non-negative.

Proposition 3.8. Assume Conjecture 1.2 in [8] holds. Then in the decomposition matrix of the principal $\Phi_{4}$-block of $\mathrm{SO}_{14}^{+}(q)$, we have $b_{1}=b_{3}=b_{4}=b_{5}=b_{8}=0, b_{6}, b_{7}, b_{10}, b_{11} \leq 2$, $b_{9} \leq 6, b_{12}, b_{13} \leq 12, b_{14} \leq 18$ and $b_{15} \leq 20$.

Proof. To obtain the upper bounds on the $b_{i}$ 's we consider the characters $Q_{w_{1}}\left[q^{3}\right]$ for $w_{1}=s_{1} s_{2} s_{3} s_{1} s_{2} s_{3} s_{5} s_{4} s_{3} s_{6} s_{5} s_{4} s_{3} s_{7} s_{6} s_{5} s_{4} s_{3}$ and $Q_{w_{2}}[1]$ for $w_{2}=s_{1} s_{2} s_{3} s_{1} s_{2} s_{3} s_{5} s_{6} s_{5} s_{7} s_{6} s_{5} s_{4}$ as well as their decomposition on the basis of PIMs.

The coefficient of $\Psi_{26}$ in $Q_{w_{1}}\left[q^{3}\right]$ is $10-14 b_{1}$. By [8, Conj. 1.2] it must be nonnegative, which forces $b_{1}=0$. The list of coefficients of $\Psi_{27}, \ldots, \Psi_{32}$ on $Q_{w_{2}}[1]$ is given by $-7 b_{3},-7 b_{4},-7 b_{5}, 17+7 b_{4}-7 b_{6}, 17-7 b_{7}$ and $7 b_{4}-7 b_{8}$. Since they must be all nonnegative, we get $b_{3}=b_{4}=b_{5}=b_{8}=0$ and $b_{6}, b_{7} \leq 2$. With these values, the coefficients of $\Psi_{33}, \ldots, \Psi_{40}$ on $Q_{w_{2}}[1]$ are

$$
\begin{aligned}
& 14+7 b_{6}+7 b_{7}-7 b_{9}, 7 b_{7}-7 b_{10}, 19-7 b_{11}, \\
& 62+7 b_{6}+7 b_{7}-7 b_{12},-9-7 b_{6}-7 b_{7}+7 b_{9}+7 b_{12}-7 b_{13}, \\
& 60-7 b_{6}-7 b_{7}+7 b_{10}+7 b_{12}-7 b_{14}+d\left(9+7 b_{6}+7 b_{7}-7 b_{9}-7 b_{12}+7 b_{13}\right), \\
& 42+21 b_{6}+21 b_{7}-21 b_{9}-7 b_{10}+14 b_{11}-21 b_{12}+21 b_{13}+7 b_{14}-7 b_{15} .
\end{aligned}
$$

¿From the first line we deduce $b_{9} \leq b_{6}+b_{7}+2, b_{10} \leq b_{7}$ and $b_{11} \leq 2$. From the second, $b_{12} \leq b_{6}+b_{7}+8$ and $b_{13} \leq-2-b_{6}-b_{7}+b_{9}+b_{12}$, so that $b_{13} \leq b_{12}$. Finally, the last two lines yield $b_{14} \leq 8-b_{6}-b_{7}+b_{10}+b_{12} \leq 16+b_{10}$ and $b_{15} \leq 6+3 b_{6}+3 b_{7}-3 b_{9}-b_{10}+$ $2 b_{11}-3 b_{12}+3 b_{13}+b_{14} \leq 16+2 b_{11}$.

3.5. Decomposition matrices for $\mathrm{SO}_{16}^{+}(q)$. Finally, we consider the three blocks of $\Phi_{4}$-defect $\Phi_{4}^{2}$ for the groups $G=\mathrm{SO}_{16}^{+}(q)$. They are again labelled by the symbol for their 4-Harish-Chandra source in a 4-split Levi subgroup of type $\mathrm{SO}_{8}^{+}(q)$.

Theorem 3.9. The $\ell$-modular decomposition matrices for the unipotent blocks of $\mathrm{SO}_{16}^{+}(q)$ of defect $\Phi_{4}^{2}$, for primes $\ell$ with $\left(q^{2}+1\right)_{\ell}>5$, are as given in Tables 4, 6, 10 and 11.

The two blocks of $\mathrm{SO}_{12}^{+}(q)$ and $\mathrm{SO}_{16}^{+}(q)$ in Table 4 are Morita equivalent, as well as the two blocks in Table 6 . 
TABLE 10. $\operatorname{SO}_{16}^{+}(q)$, block $\left(\begin{array}{l}12 \\ 03\end{array}\right),\left(q^{2}+1\right)_{\ell}>5$

\begin{tabular}{|c|c|c|c|c|c|c|c|c|c|c|c|c|c|}
\hline $1^{2} .6$ & $\frac{1}{2} \Phi_{8} \Phi_{10}$ & 1 & & & & & & & & & & & \\
\hline 2.51 & $\frac{1}{2} q \Phi_{5} \Phi_{8} \Phi_{14}$ & . 1 & & & & & & & & & & & \\
\hline 3.41 & $\frac{1}{2} q^{2} \Phi_{2}^{4} \Phi_{6} \Phi_{10} \Phi_{14}$ & 11 & 1 & & & & & & & & & & \\
\hline 2.42 & $\frac{1}{2} q^{3} \Phi_{3}^{2} \Phi_{8} \Phi_{10} \Phi_{14}$ & . 1 & 1 & 1 & & & & & & & & & \\
\hline $1^{2} .3^{2}$ & $\frac{1}{2} q^{6} \Phi_{5} \Phi_{6} \Phi_{8} \Phi_{10} \Phi_{14}$ & 1 & 1 & . & 1 & & & & & & & & \\
\hline $.42^{2}$ & $\frac{1}{2} q^{7} \Phi_{8}^{2} \Phi_{10} \Phi_{14}$ & . & . & 1 & . & 1 & & & & & & & \\
\hline$D_{4}: 1^{2} .2$ & $\frac{1}{2} q^{8} \Phi_{1}^{4} \Phi_{3}^{2} \Phi_{5} \Phi_{6} \Phi_{14}$ & . & . & . & . & . & 1 & & & & & & \\
\hline $2.2^{3}$ & $\frac{1}{2} q^{10} \Phi_{5} \Phi_{6} \Phi_{8} \Phi_{10} \Phi_{14}$ & . & 1 & 1 & . & 1 & . & 1 & & & & & \\
\hline$D_{4}: 2.1^{2}$ & $\frac{1}{2} q^{10} \Phi_{1}^{4} \Phi_{3}^{2} \Phi_{5} \Phi_{10}$ & . & . & . & . & . & . & . & 1 & & & & \\
\hline $.3^{2} 1^{2}$ & $\frac{1}{2} q^{11} \Phi_{8}^{2} \Phi_{10} \Phi_{14}$ & . & . & . & 1 & . & . & . & . & 1 & & & \\
\hline $1^{2} .2^{2} 1^{2}$ & $\frac{1}{2} q^{15} \Phi_{3}^{2} \Phi_{8} \Phi_{10} \Phi_{14}$ & . & 1 & . & 1 & . & . & . & . & 1 & 1 & & \\
\hline $1^{3} \cdot 21^{3}$ & $\frac{1}{2} q^{18} \Phi_{2}^{4} \Phi_{6} \Phi_{10} \Phi_{14}$ & . & 1 & . & . & . & . & 1 & . & . & 1 & 1 & \\
\hline $1^{2} .21^{4}$ & $\frac{1}{2} q^{21} \Phi_{5} \Phi_{8} \Phi_{14}$ & . & . & . & . & . & 2 & . & . & 1 & 1 & 1 & 1 \\
\hline $1^{6} .2$ & $\frac{1}{2} q^{28} \Phi_{8} \Phi_{10}$ & . & . & . & . & 1 & & 1 & 2 & & & 1 & 1 \\
\hline
\end{tabular}

Here, all character degrees have been divided by $q^{3} \Phi_{4}^{2} \Phi_{7} \Phi_{12}$.

TABLE 11. $\mathrm{SO}_{16}^{+}(q)$, block $\left(\begin{array}{l}23 \\ 01\end{array}\right),\left(q^{2}+1\right)_{\ell}>5$

\begin{tabular}{|c|c|c|c|c|c|c|c|c|c|c|c|c|c|}
\hline .62 & $\frac{1}{2} \Phi_{5} \Phi_{8}$ & 1 & & & & & & & & & & & \\
\hline .53 & $\frac{1}{2} q \Phi_{7} \Phi_{8} \Phi_{10}$ & 11 & & & & & & & & & & & \\
\hline$D_{4}: 2^{2}$. & $\frac{1}{2} q^{2} \Phi_{1}^{4} \Phi_{3} \Phi_{5} \Phi_{7}$ & . . & 1 & & & & & & & & & & \\
\hline $2^{2} .4$ & $\frac{1}{2} q^{3} \Phi_{5} \Phi_{6}^{2} \Phi_{7} \Phi_{8}$ & 1 & . & 1 & & & & & & & & & \\
\hline $2^{2} .31$ & $\frac{1}{2} q^{6} \Phi_{3} \Phi_{5} \Phi_{7} \Phi_{8} \Phi_{10}$ & 11 & . & 1 & 1 & & & & & & & & \\
\hline $1^{2} .41^{2}$ & $\frac{1}{2} q^{7} \Phi_{5} \Phi_{7} \Phi_{8}^{2}$ & . . & . & 1 & . & 1 & & & & & & & \\
\hline $21.31^{2}$ & $\frac{1}{2} q^{8} \Phi_{2}^{4} \Phi_{3} \Phi_{6}^{2} \Phi_{7} \Phi_{10}$ & . & . & 1 & 1 & 1 & 1 & & & & & & \\
\hline $1.41^{3}$ & $\frac{1}{2} q^{10} \Phi_{2}^{4} \Phi_{5} \Phi_{6}^{2} \Phi_{10}$ & . & . & . & . & 1 & . & 1 & & & & & \\
\hline $21^{2} .2^{2}$ & $\frac{1}{2} q^{10} \Phi_{3} \Phi_{5} \Phi_{7} \Phi_{8} \Phi_{10}$ & . 1 & . & . & 1 & . & 1 & . & 1 & & & & \\
\hline $2.31^{3}$ & $\frac{1}{2} q^{11} \Phi_{5} \Phi_{7} \Phi_{8}^{2}$ & . . & 2 & . & . & 1 & 1 & 1 & . & 1 & & & \\
\hline $1^{4} .2^{2}$ & $\frac{1}{2} q^{15} \Phi_{5} \Phi_{6}^{2} \Phi_{7} \Phi_{8}$ & . & 2 & . & . & . & 1 & & 1 & 1 & 1 & & \\
\hline$D_{4}: .2^{2}$ & $\frac{1}{2} q^{18} \Phi_{1}^{4} \Phi_{3} \Phi_{5} \Phi_{7}$ & . & $\cdot$ & . & . & . & . & . & & . & . & 1 & \\
\hline $.2^{3} 1^{2}$ & $\frac{1}{2} q^{21} \Phi_{7} \Phi_{8} \Phi_{10}$ & . 1 & & & . & . & . & & 1 & . & . & . & 1 \\
\hline $.2^{2} 1^{4}$ & $\frac{1}{2} q^{28} \Phi_{5} \Phi_{8}$ & . & . & . & . & . & . & & 1 & . & 1 & 2 & \\
\hline
\end{tabular}

Here, all character degrees have been divided by $q^{3} \Phi_{4}^{2} \Phi_{12} \Phi_{14}$.

Proof. Harish-Chandra induction sends the fourteen PIMs in the principal block of $\mathrm{SO}_{12}^{+}(q)$ to the fourteen listed projectives of the first block of $\mathrm{SO}_{16}^{+}(q)$ in Table 4 , and it sends irreducible characters to irreducible characters. Thus, by [2, Thm. 0.2] those two blocks are 
Morita equivalent. Exactly the same assertions hold for the third block of $\mathrm{SO}_{12}^{+}(q)$ and the fourth block of $\mathrm{SO}_{16}^{+}(q)$ in Table 6 .

In the second block of $G,(\mathrm{HCi})$ yields all columns in Table 10, except for the second one. This is then obtained from the projectives $\Psi_{1}+\Psi_{2}$ and $\Psi_{2}+\Psi_{4}$ via (Sum).

In the third block, we obtain all $\Psi_{i}$ in Table 11 for $i \notin\{1,5,6,9\}$. Then, using (Sum) $\Psi_{4}+\Psi_{6}$ and $\Psi_{6}+\Psi_{7}$ give $\Psi_{6}, \Psi_{2}+\Psi_{9}$ and $\Psi_{7}+\Psi_{9}+\Psi_{11}$ give $\Psi_{9}, \Psi_{4}+\Psi_{5}$ and $\Psi_{5}+\Psi_{9}$ give $\Psi_{5}$, and finally $\Psi_{1}+\Psi_{2}$ and $\Psi_{1}+\Psi_{5}$ give $\Psi_{1}$.

\section{Decomposition MAtrices FOR $E_{6}(q)$}

We now turn to decomposition matrices for the exceptional Lie type groups. We first consider $G=E_{6}(q)$ for primes $\ell \mid \Phi_{4}(q)$ in which case the Sylow $\ell$-subgroups are abelian homocyclic of rank 2. Note that again we do not need and will not specify the isogeny type of $G$, since the decomposition numbers of the unipotent characters will not depend on such a choice. The group $E_{6}(q)$ has ten unipotent blocks of $\ell$-defect zero, one of cyclic defect and the principal block containing 16 unipotent characters.

Here, the decomposition matrix of the principal block has been determined by Miyachi [22, Thm. 37] except for three missing entries, which coincide with entries of the decomposition matrix for $D_{4}(q)$, again under the assumption that $q$ is a power of a good prime. We give an independent proof of his result, valid for all prime powers $q$, and find the remaining entries using Theorem 3.1;

Proposition 4.1. Let $\ell$ be a prime. Then the $\ell$-modular decomposition matrices for the unipotent blocks of $E_{6}(q)$ of positive defect, for $\left(q^{2}+1\right)_{\ell}>5$, are as given in Tables 12 and 13. In particular, the three undetermined entries in the $\ell$-modular decomposition matrix of $E_{6}(q)$ in [22, Thm. 37] are all equal to 2.

Proof. The Brauer tree for the block with cyclic defect is easily determined. We will construct PIMs $\Psi_{1}, \ldots, \Psi_{16}$ with unipotent parts as given by the columns of Table 12 .

The PIMs in the principal series can be read off from the $\Phi_{4}$-modular decomposition matrix of the Iwahori-Hecke algebra of type $E_{6}$ given in [14, Tab. 7.13]. Note that by [15, Thm. 3.10] this agrees with the $\ell$-modular decomposition matrix whenever $\ell \geq 5$ (since 5 is a good prime for $E_{6}$ and 20 does not divide any degree of $E_{6}$ ). So we have columns $i$ in Table 12 for $i \in\{1,2,3,4,6,7,8,9\}$. Furthermore, $\Psi_{11}$ and $\Psi_{13}$ are Harish-Chandra induced from Levi subgroups of types $D_{5}$ and $A_{5}$.

The Hecke algebra for the ordinary cuspidal unipotent character of $D_{4}$ is of type $A_{2}$ with parameter $q$, thus remains semisimple modulo $\ell$. Now Harish-Chandra induction from the Levi subgroup $L$ of type $D_{5}$ yields projective characters $\Psi_{5}+\Psi_{10}$ and $\Psi_{10}+\Psi_{14}$. Both of these must be the sum of two projective characters. Using ( $\mathrm{HCr}$ ) we find that $\Psi_{5}, \Psi_{10}, \Psi_{14}$ are the only subsums which can be projective.

Since all other Harish-Chandra series have been accounted for, and $G$ cannot have cuspidal Brauer characters by (Csp), the missing three PIMs must lie in the series of the cuspidal Brauer character $\rho_{.1^{4}}$ of $D_{4}$. Its relative Weyl group is the symmetric group $\mathfrak{S}_{3}$, so the corresponding Hecke algebra must be semisimple. Now Harish-Chandra induction yields $\Psi_{12}+\Psi_{15}$ and $\Psi_{15}+\Psi_{16}$, and via ( $\mathrm{HCr}$ ) there is a unique way for each of these to split into sums of two non-zero projective characters. This completes the construction of $\Psi_{1}, \ldots, \Psi_{16}$ and thus the proof. 
TABLE 12. $E_{6}(q),\left(q^{2}+1\right)_{\ell}>5$

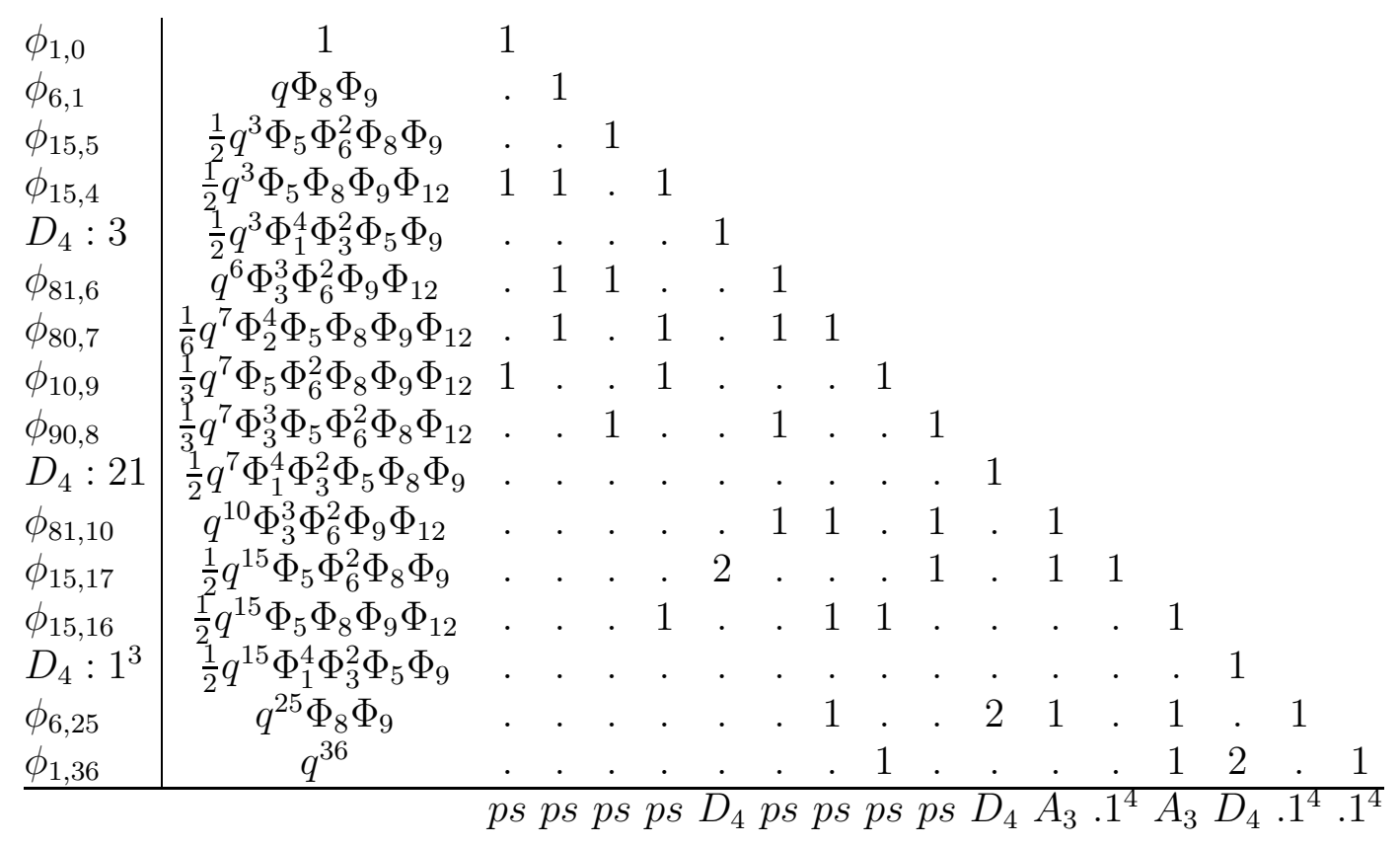

TABLE 13. $E_{6}(q)$, block of defect $1,2 \neq \ell \mid\left(q^{2}+1\right)$

$$
\phi_{20,2} \underset{p s}{-} \phi_{60,5}-\phi_{6 s} \phi_{60,11}-\phi_{20,20}-A_{3}
$$

\section{Decomposition matrices For $E_{7}(q)$}

We next consider the four unipotent $\Phi_{4}$-blocks of $E_{7}(q)$ of positive defect (see [3, Tab. 2]), which we name by their 4-Harish-Chandra sources in a Levi subgroup of type $A_{1}(q)^{3}$.

Theorem 5.1. The $\ell$-modular decomposition matrices for the unipotent blocks of $E_{7}(q)$ of positive defect for primes $\ell$ with $\left(q^{2}+1\right)_{\ell}>5$ are as given in Tables 14417 .

Proof. For the principal block, all columns but the sixth are obtained by $(\mathrm{HCi})$. The projectives $\Psi_{6}+\Psi_{7}$ and $\Psi_{6}+\Psi_{10}$ then yield $\Psi_{6}$ via (Sum).

For the second block, all but the ninth column are gotten by (HCi) and then $\Psi_{9}+\Psi_{10}$ and $\Psi_{9}+\Psi_{12}$ give $\Psi_{9}$.

For the third block, all PIMs are Harish-Chandra induced. Finally, all projectives in the fourth block except for the fifth come from (HCi). The projectives $\Psi_{5}+\Psi_{7}$ and $\Psi_{5}+\Psi_{10}$ then give $\Psi_{5}$ via (Sum). Then ( $\mathrm{HCr}$ ) shows that all of these projectives are indecomposable. 
TABLE $14 . E_{7}(q)$, block $2 \otimes 2 \otimes 2,\left(q^{2}+1\right)_{\ell}>5$

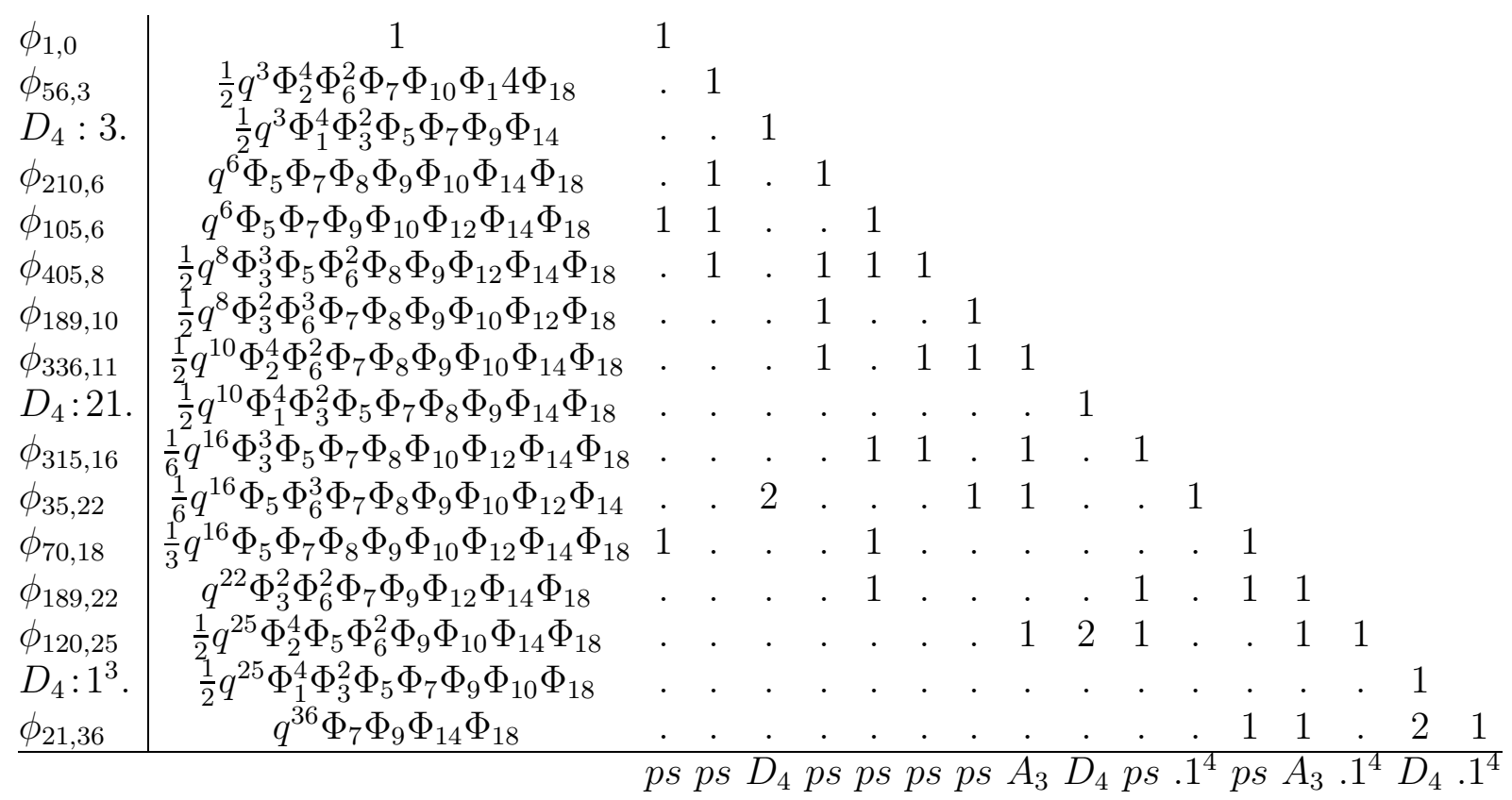

TABLE $15 . E_{7}(q)$, block $2 \otimes 2 \otimes 1^{2},\left(q^{2}+1\right)_{\ell}>5$

\begin{tabular}{|c|c|c|c|c|c|c|c|c|c|c|c|c|c|}
\hline$\phi_{7,1}$ & $\begin{array}{c}q \Phi_{7} \Phi_{12} \Phi_{14} \\
\underline{1} g^{4} \Phi_{-} \Phi_{0} \Phi_{1} \Phi_{1} \Phi_{1}\end{array}$ & 1 & & & & & & & & & & & \\
\hline$\phi_{15,7}$ & $\frac{1}{2} q^{4} \Phi_{5} \Phi_{8} \Phi_{9} \Phi_{10} \Phi_{12} \Phi_{14} \Phi_{18}$ & 11 & & & & & & & & & & & \\
\hline$\phi_{105,5}$ & $\frac{1}{2} q^{4} \Phi_{5} \Phi_{7} \Phi_{8} \Phi_{9} \Phi_{10} \Phi_{12} \Phi_{18}$ & . & 1 & & & & & & & & & & \\
\hline$\phi_{189,7}$ & $q^{7} \Phi_{3}^{2} \Phi_{6}^{2} \Phi_{7} \Phi_{9} \Phi_{12} \Phi_{14} \Phi_{18}$ & . & 1 & 1 & & & & & & & & & \\
\hline$\phi_{280,8}$ & $\frac{1}{2} q^{7} \Phi_{2}^{4} \Phi_{5} \Phi_{6}^{3} \Phi_{7} \Phi_{10} \Phi_{12} \Phi_{14} \Phi_{18}$ & 1 & 1 & 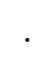 & 1 & & & & & & & & \\
\hline$D_{4}: 2.1$ & $\frac{1}{2} q^{7} \Phi_{1}^{4} \Phi_{3}^{3} \Phi_{5} \Phi_{7} \Phi_{9} \Phi_{10} \Phi_{12} \Phi_{14}$ & . & . & . & . & 1 & & & & & & & \\
\hline$\phi_{378,9}$ & $q^{9} \Phi_{3}^{2} \Phi_{6}^{2} \Phi_{7} \Phi_{8} \Phi_{9} \Phi_{12} \Phi_{14} \Phi_{18}$ & . & 1 & 1 & 1 & 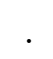 & 1 & & & & & & \\
\hline$\phi_{210,13}$ & $q^{13} \Phi_{5} \Phi_{7} \Phi_{8} \Phi_{9} \Phi_{10} \Phi_{12} \Phi_{14} \Phi_{18}$ & 11 & . & . & 1 & 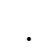 & . & 1 & & & & & \\
\hline$\phi_{105,15}$ & $q^{15} \Phi_{5} \Phi_{7} \Phi_{9} \Phi_{10} \Phi_{12} \Phi_{14} \Phi_{18}$ & . & . & 1 & . & . & 1 & $\cdot$ & 1 & & & & \\
\hline$\phi_{216,16}$ & $\frac{1}{2} q^{15} \Phi_{2}^{4} \Phi_{3}^{2} \Phi_{6}^{3} \Phi_{9} \Phi_{10} \Phi_{12} \Phi_{14} \Phi_{18}$ & . & . & . & 1 & . & 1 & 1 & & 1 & & & \\
\hline$D_{4}: 1^{2} .1$ & $\frac{1}{2} q^{15} \Phi_{1}^{4} \Phi_{3}^{3} \Phi_{5} \Phi_{6}^{2} \Phi_{7} \Phi_{9} \Phi_{12} \Phi_{18}$ & . & . & . & . & . & . & $\cdot$ & & & 1 & & \\
\hline$\phi_{35,31}$ & $\frac{1}{2} q^{30} \Phi_{5} \Phi_{7} \Phi_{8} \Phi_{12} \Phi_{14} \Phi_{18}$ & 1 & . & . & . & . & . & 1 & . & $\cdot$ & . & 1 & \\
\hline$\phi_{21,33}$ & $\frac{1}{2} q^{30} \Phi_{7} \Phi_{8} \Phi_{9} \Phi_{10} \Phi_{12} \Phi_{14}$ & . & . & . & . & 2 & 1 & . & 1 & 1 & . & . & 1 \\
\hline$\phi_{27,37}$ & $q^{37} \Phi_{3}^{2} \Phi_{6}^{2} \Phi_{9} \Phi_{12} \Phi_{18}$ & & . & & & & . & 1 & & 1 & 2 & 1 & 1 \\
\hline
\end{tabular}

\section{Decomposition matrices for $E_{8}(q)$}

Finally, we determine the decomposition matrices of the unipotent $\Phi_{4}$-blocks of $G=$ $E_{8}(q)$ of non-maximal defect. Since 5 is a bad prime for $G$, and hence the basic set results do not apply in this case, we assume that $\ell \neq 5$ throughout. There are four unipotent 
TABLE $16 . E_{7}(q)$, block $2 \otimes 1^{2} \otimes 1^{2},\left(q^{2}+1\right)_{\ell}>5$

\begin{tabular}{|c|c|c|c|c|c|c|c|c|c|c|c|c|c|}
\hline$\phi_{27,2}$ & $q^{2} \Phi_{3}^{2} \Phi_{6}^{2} \Phi_{9} \Phi_{12} \Phi_{18}$ & 1 & & & & & & & & & & & \\
\hline$\phi_{35,4}$ & $\frac{1}{2} q^{3} \Phi_{5} \Phi_{7} \Phi_{8} \Phi_{12} \Phi_{14} \Phi_{18}$ & 11 & & & & & & & & & & & \\
\hline$\phi_{21,6}$ & $\frac{1}{2} q^{3} \Phi_{7} \Phi_{8} \Phi_{9} \Phi_{10} \Phi_{12} \Phi_{14}$ & . . & 1 & & & & & & & & & & \\
\hline$\phi_{216,9}$ & $\frac{1}{2} q^{8} \Phi_{2}^{4} \Phi_{3}^{2} \Phi_{6}^{3} \Phi_{9} \Phi_{10} \Phi_{12} \Phi_{14} \Phi_{18}$ & 1 & 1 & 1 & & & & & & & & & \\
\hline$D_{4}: 1.2$ & $\frac{1}{2} q^{8} \Phi_{1}^{4} \Phi_{3}^{3} \Phi_{5} \Phi_{6}^{2} \Phi_{7} \Phi_{9} \Phi_{12} \Phi_{18}$ & . & . & · & 1 & & & & & & & & \\
\hline$\phi_{210,10}$ & $q^{10} \Phi_{5} \Phi_{7} \Phi_{8} \Phi_{9} \Phi_{10} \Phi_{12} \Phi_{14} \Phi_{18}$ & 11 & . & 1 & & 1 & & & & & & & \\
\hline$\phi_{105,12}$ & $q^{12} \Phi_{5} \Phi_{7} \Phi_{9} \Phi_{10} \Phi_{12} \Phi_{14} \Phi_{18}$ &. & 1 & & & $\cdot$ & 1 & & & & & & \\
\hline$\phi_{378,14}$ & $q^{14} \Phi_{3}^{2} \Phi_{6}^{2} \Phi_{7} \Phi_{8} \Phi_{9} \Phi_{12} \Phi_{14} \Phi_{18}$ & . & 1 & 1 & & . & 1 & 1 & & & & & \\
\hline$\phi_{280,17}$ & $\frac{1}{2} q^{16} \Phi_{2}^{4} \Phi_{5} \Phi_{6}^{3} \Phi_{7} \Phi_{10} \Phi_{12} \Phi_{14} \Phi_{18}$ & . & . & 1 & & 1 & & 1 & 1 & & & & \\
\hline$D_{4}: 1.1^{2}$ & $\frac{1}{2} q^{16} \Phi_{1}^{4} \Phi_{3}^{3} \Phi_{5} \Phi_{7} \Phi_{9} \Phi_{10} \Phi_{12} \Phi_{14}$ & . & . & & & & . & & & 1 & & & \\
\hline$\phi_{189,20}$ & $q^{20} \Phi_{3}^{2} \Phi_{6}^{2} \Phi_{7} \Phi_{9} \Phi_{12} \Phi_{14} \Phi_{18}$ & . & . & & & . & 1 & 1 & . & . & 1 & & \\
\hline$\phi_{15,28}$ & $\frac{1}{2} q^{25} \Phi_{5} \Phi_{8} \Phi_{9} \Phi_{10} \Phi_{12} \Phi_{14} \Phi_{18}$ & 1 & 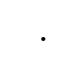 & & & 1 & . & & . & . & & 1 & \\
\hline$\phi_{105,26}$ & $\frac{1}{2} q^{25} \Phi_{5} \Phi_{7} \Phi_{8} \Phi_{9} \Phi_{10} \Phi_{12} \Phi_{18}$ & . & . & & 2 & & . & 1 & 1 & $\cdot$ & 1 & & 1 \\
\hline$\phi_{7,46}$ & $q^{46} \Phi_{7} \Phi_{12} \Phi_{14}$ & . & . & & & 1 & . & . & 1 & 2 & & 1 & . \\
\hline
\end{tabular}

TABLE $17 . E_{7}(q)$, block $1^{2} \otimes 1^{2} \otimes 1^{2},\left(q^{2}+1\right)_{\ell}>5$

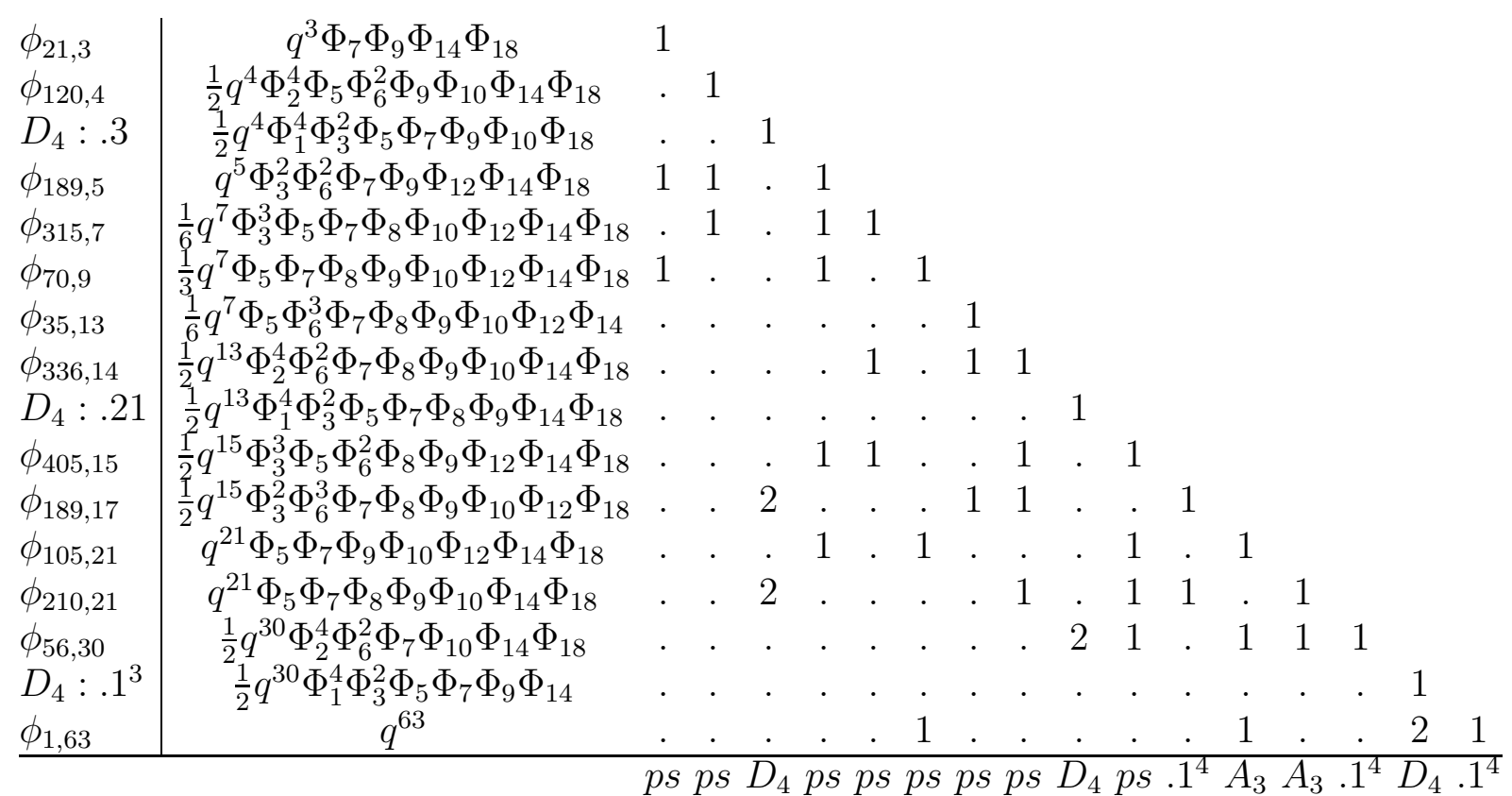

$\ell$-blocks of $\Phi_{4}$-defect two, see [3, Tab. 2]. These are labelled by the four $\Phi_{4}$-cuspidal unipotent characters of the $\Phi_{4}$-split Levi subgroup of type $D_{4}$.

Theorem 6.1. The $\ell$-modular decomposition matrices for the unipotent blocks of $E_{8}(q)$ of defect $\Phi_{4}^{2}$ for primes $\ell>5$ dividing $q^{2}+1$ are as given in Tables 18 21. 
TABLE 18. $E_{8}(q)$, block $\left(\begin{array}{l}3 \\ 1\end{array}\right), 5<\ell \mid\left(q^{2}+1\right)$

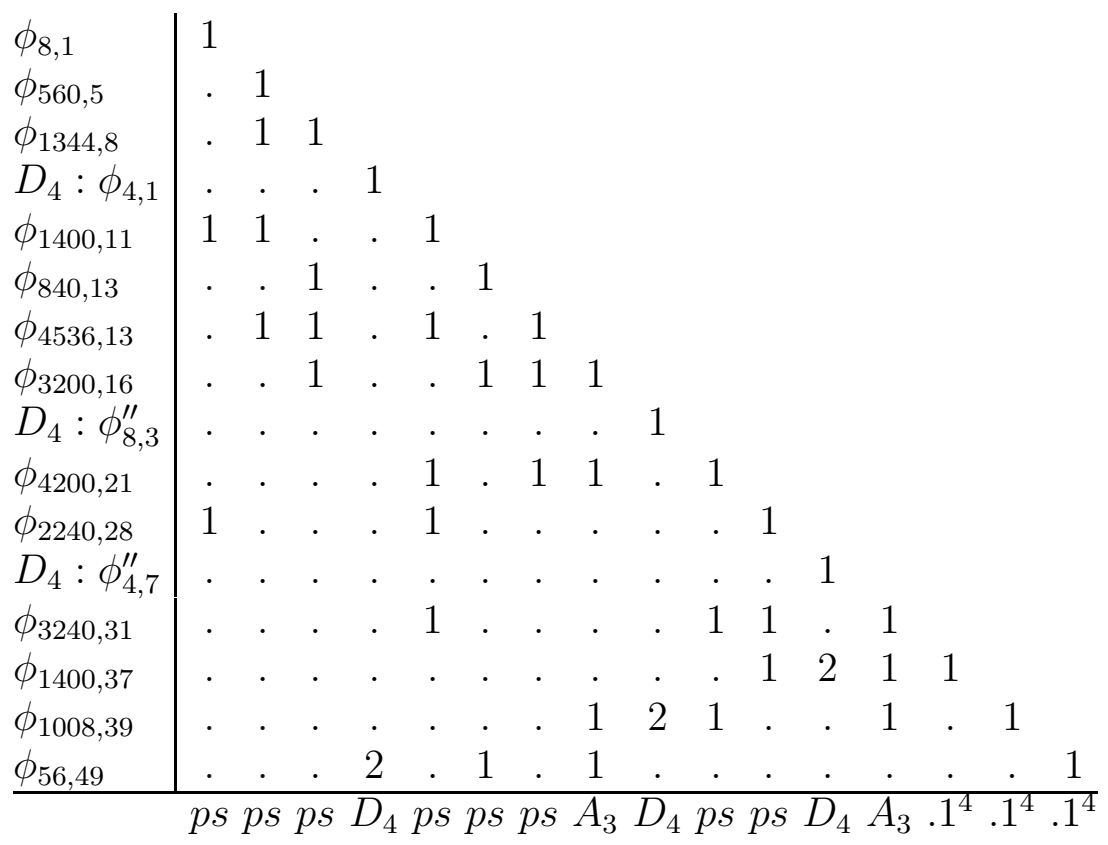

TABLE 19. $E_{8}(q)$, block $\left(\begin{array}{l}12 \\ 03\end{array}\right), 5<\ell \mid\left(q^{2}+1\right)$

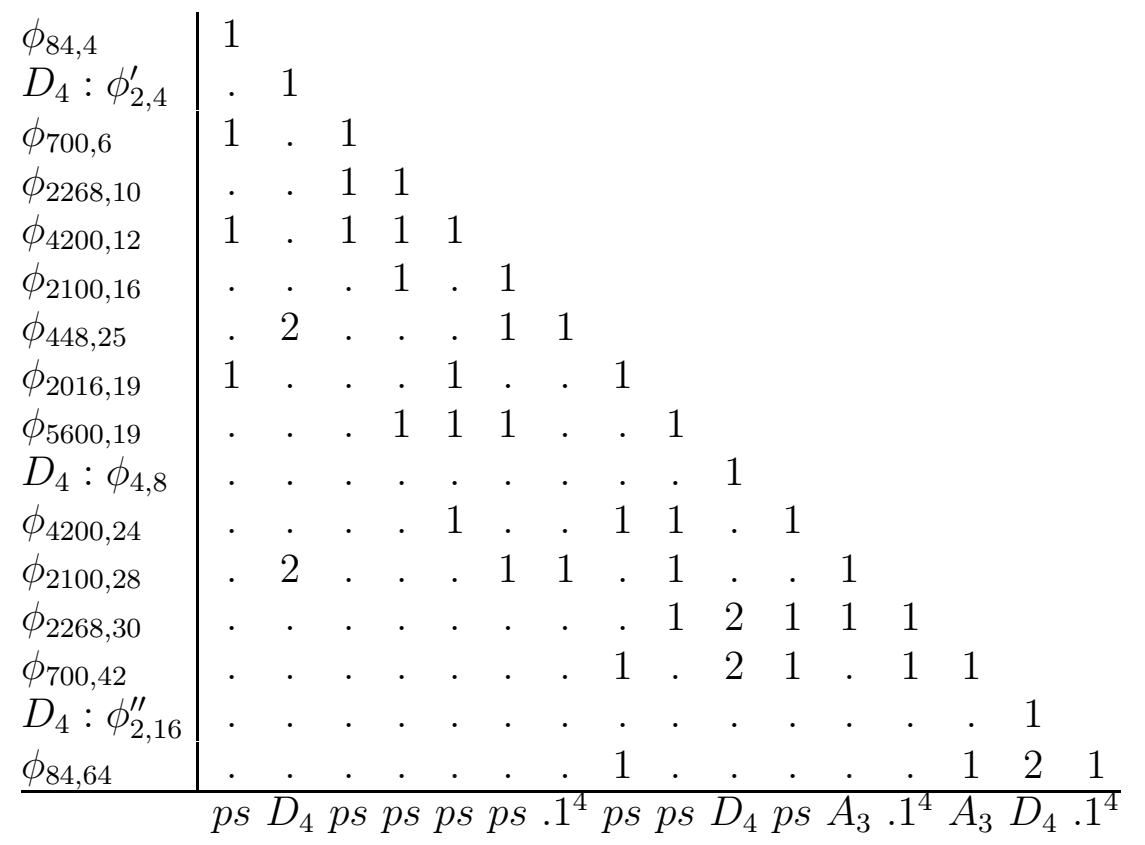

Proof. In the block above $\left(\begin{array}{l}3 \\ 1\end{array}\right)$, all columns $\Psi_{i}$ except for $i \in\{7,9,10,15\}$ are obtained by (HCi). Using (Sum) the projectives $\Psi_{1}+\Psi_{2}+\Psi_{7}$ and $\Psi_{7}+\Psi_{11}$ yield $\Psi_{7}, \Psi_{4}+\Psi_{9}$ and 
TABLE 20. $E_{8}(q)$, block $\left(\begin{array}{l}23 \\ 01\end{array}\right), 5<\ell \mid\left(q^{2}+1\right)$

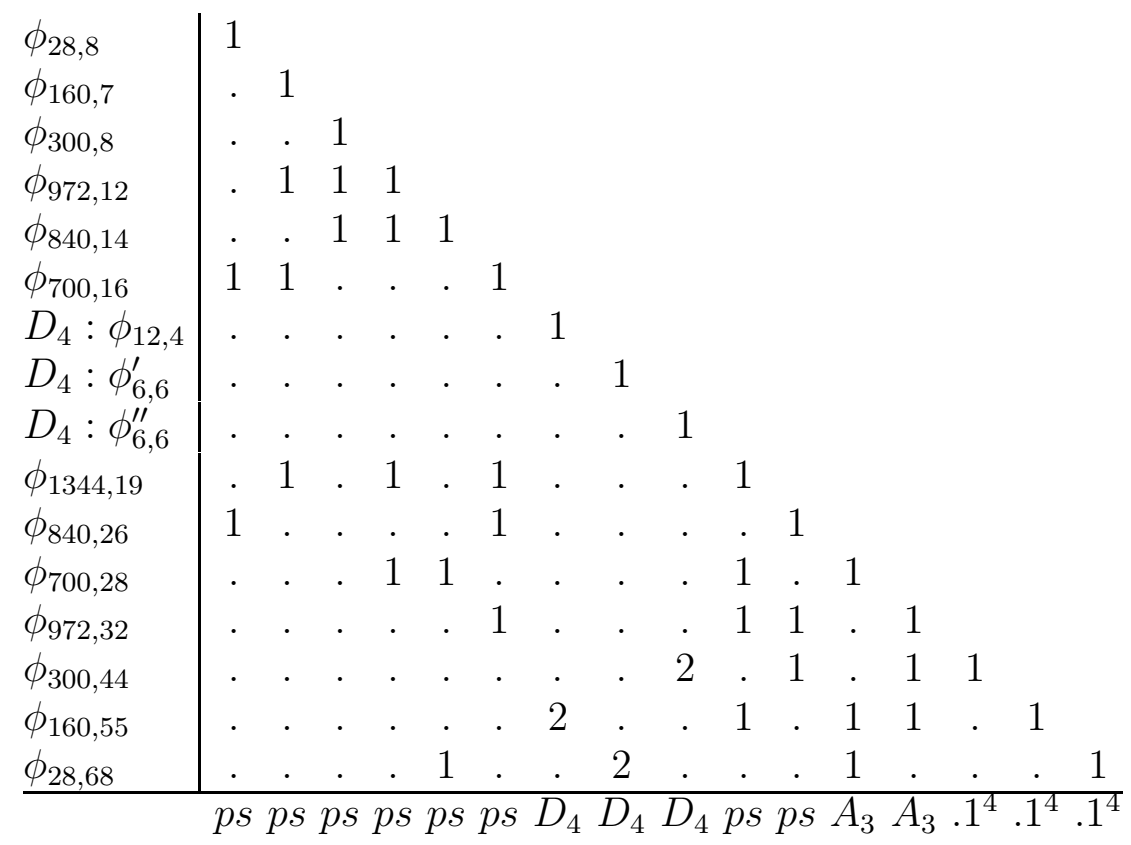

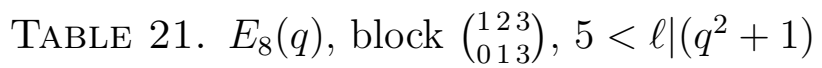

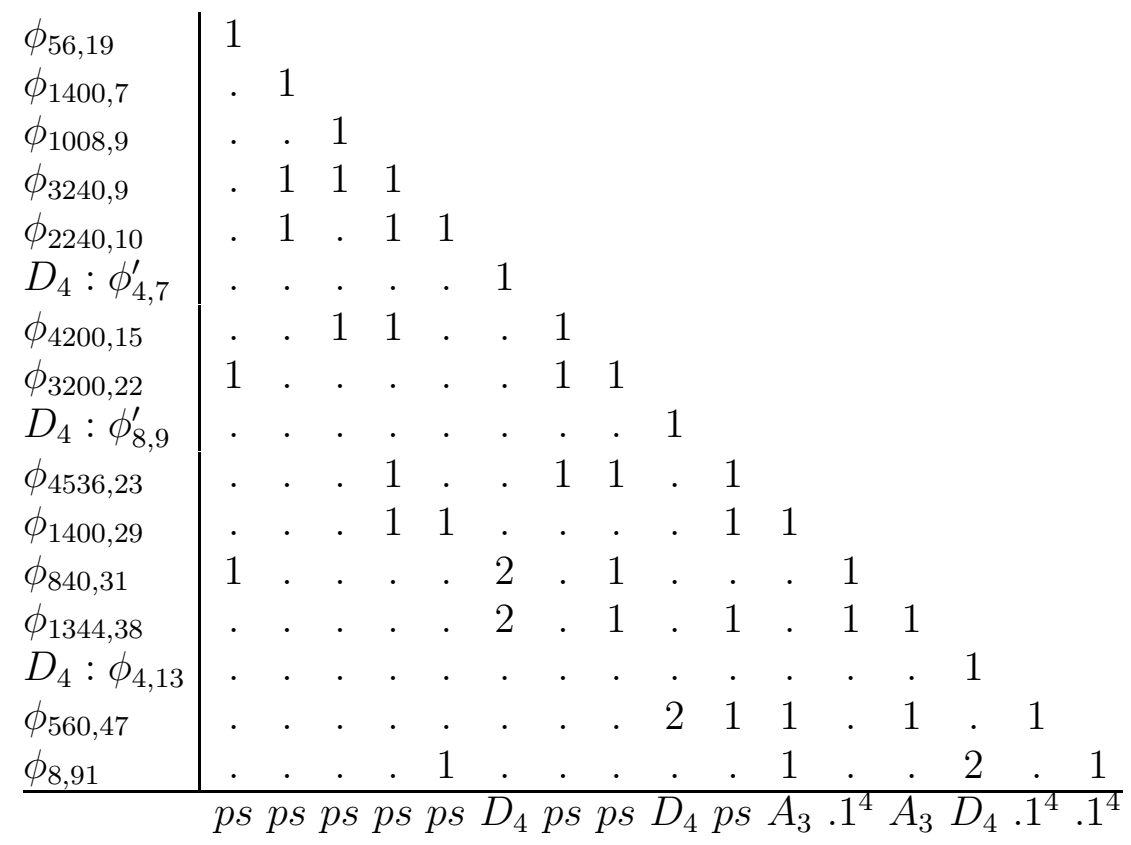

$\Psi_{9}+\Psi_{12}$ yield $\Psi_{9}, \Psi_{6}+2 \Psi_{7}+\Psi_{10}$ and $\Psi_{10}+\Psi_{11}$ yield $\Psi_{10}$, and $\Psi_{11}+\Psi_{15}$ and $\Psi_{14}+\Psi_{15}$ yield $\Psi_{15}$. 
In the block above $\left(\begin{array}{l}12 \\ 03\end{array}\right),(\mathrm{HCi})$ yields all columns $\Psi_{i}$ except for indices $i \in\{3,6,11\}$. Here, the projectives $\Psi_{11}+\Psi_{12}$ and $\Psi_{11}+\Psi_{14}$ yield $\Psi_{11}, \Psi_{3}+\Psi_{5}+\Psi_{11}$ and $2 \Psi_{3}$ yield $\Psi_{3}$, and $\Psi_{6}+2 \Psi_{9}$ and $2 \Psi_{6}$ yield $\Psi_{6}$.

In the block above $\left(\begin{array}{l}23 \\ 01\end{array}\right)$, ( $\mathrm{HCi}$ ) yields the columns $\Psi_{i}$ with $i \in\{3,4,6,11,12,13\}$. The information obtained in this way does not seem to yield strong enough conditions to determine the decomposition matrix completely. So here we use in addition the decomposition matrix of the Hecke algebra of type $E_{8}$. In characteristic zero this can be found in [14, Tab. 7.15]. By [15, Thm. 3.10] this agrees with the decomposition matrix in characteristic $\ell$ for all $\ell \geq 7$. This yields in addition the principal series PIMs $\Psi_{i}$ with $i \in\{1,2,5,10\}$. To construct the missing projectives, let us first consider the characters in the Harish-Chandra series of the ordinary cuspidal character of a Levi subgroup of type $D_{4}$. Here, the relative Weyl group $W$ has type $F_{4}$, and the Hecke algebra $H$ has parameters $q^{4}, q$. But then all characters of $H$ relevant for our block lie in semisimple blocks of $H$ for all $\ell \geq 5$ by [1, Thm. 3.13]. Thus, the decomposition of induced projectives from a Levi subgroup of type $E_{7}$ can be read off from the character table of $W$. In the third block, we find $\Psi_{7}+\Psi_{8}, \Psi_{7}+\Psi_{9}$, which should have a projective summand in common. The only splitting of these projectives compatible with ( $\mathrm{HCr})$ is as given. This accounts for the projectives $\Psi_{7}, \Psi_{8}, \Psi_{9}$ in the $D_{4}$-series. Finally, we consider the characters above the cuspidal character $.1^{4}$ of the Levi subgroup of type $D_{4}$. Here again, the relative Weyl group has type $F_{4}$; the parameters of the corresponding Hecke algebra are already determined locally inside $D_{5}$ and $D_{4} A_{1}$ to be the same as for the ordinary cuspidal character considered above. So again by [1, Thm. 3.13], all characters in the $.1^{4}$-Harish-Chandra series correspond to blocks of $H$ of defect 0 . Harish-Chandra induction from $E_{7}$ yields $\Psi_{14}+\Psi_{15}$ and $\Psi_{15}+\Psi_{16}$, with a common summand. Visible, the only possible splitting is as claimed.

Finally, in the block indexed by $\left(\begin{array}{l}123 \\ 013\end{array}\right)$, ( $\left.\mathrm{HCi}\right)$ yields all columns $\Psi_{i}$ except for $i \in$ $\{2,3,7\}$. The projectives $\Psi_{5}+\Psi_{7}+\Psi_{10}$ and $2 \Psi_{7}+\Psi_{8}$ yield $\Psi_{7}, \Psi_{2}+\Psi_{7}$ and $2 \Psi_{2}+\Psi_{4}$ yield $\Psi_{2}$, and $\Psi_{1}+\Psi_{3}+2 \Psi_{7}$ and $\Psi_{2}+\Psi_{3}$ give $\Psi_{3}$.

It is now a routine computation using ( $\mathrm{HCr}$ ) to check that none of the projectives constructed above can be decomposable.

6.1. The $\Phi_{4}$-blocks in untwisted groups. In the following table, we have collected some numerical information on the various $\Phi_{4}$-blocks of defect $\Phi_{4}^{2}$ and $\Phi_{4}^{3}$ whose decomposition matrices we have determined: the relative Weyl group $W_{G}(b)$ of the block (see [3. Tab. 1]) and the distribution of its Brauer characters into Harish-Chandra series:

Remark 6.2. (a) It emerges that the distribution into modular Harish-Chandra series in all examples considered only depends on the relative Weyl group.

(b) In addition to the first block of $\mathrm{SO}_{12}^{+}(q)$ and the first block of $\mathrm{SO}_{16}^{+}(q)$, and the third block of $\mathrm{SO}_{12}^{+}(q)$ and the fourth block of $\mathrm{SO}_{16}^{+}(q)$, which form Morita equivalent pairs by Theorem [3.9, the following four pairs of blocks have identical decomposition matrices (after suitably reordering the characters): the principal block of $E_{6}$ and the 3rd block of $E_{8}$; the 2 nd and the 3 rd block of $E_{7}$; the first blocks of $E_{7}$ and of $E_{8}$; the 4 th blocks of $E_{7}$ and of $E_{8}$. It is claimed (without proof) in [22, Rem. 34] that the first listed pair of blocks are in fact Morita equivalent. It would be interesting to see whether this is true for all pairs mentioned above. 
TABLE 22. HC-series in $\Phi_{4}$-blocks of defect $\Phi_{4}^{2}$ and $\Phi_{4}^{3}$

\begin{tabular}{cc|cc|ccccccc}
$G$ & $b$ & $W_{G}(b)$ & $|\operatorname{IBr}(b)|$ & $p s$ & $A_{3}$ & $D_{3}$ & $D_{4}$ & $.1^{4}$ & $A_{3} D_{3}$ & $c$ \\
\hline$D_{4}$ & & $G(4,2,2)$ & 10 & 5 & 2 & 1 & 1 & 1 & & \\
$D_{6}$ & 2 & & & 5 & 2 & 1 & 1 & 1 & & \\
\hline$D_{5}$ & & $G(4,1,2)$ & 14 & 7 & 1 & 2 & 2 & 2 & & \\
$D_{6}$ & 1,3 & & & 7 & 1 & 2 & 2 & 2 & & \\
$D_{7}$ & 2 & & & 7 & 1 & 2 & 2 & 2 & & \\
$D_{8}$ & $1-4$ & & & 7 & 1 & 2 & 2 & 2 & & \\
$E_{7}$ & 2,3 & & & 7 & & 3 & 2 & 2 & & \\
\hline$E_{6}$ & & $G_{8}$ & 16 & 8 & & 2 & 3 & 3 & & \\
$E_{7}$ & 1,4 & & & 8 & & 2 & 3 & 3 & & \\
$E_{8}$ & $1-4$ & & & 8 & & 2 & 3 & 3 & & \\
\hline$D_{7}$ & 1 & $G(4,1,3)$ & 40 & 15 & 3 & 5 & 6 & 6 & 1 & 4
\end{tabular}

Note that the series $A_{3}$ and $D_{3}$ fuse in $E_{6}$ (and hence in $E_{7}$ and $E_{8}$ ).

(c) The decomposition matrix for $\mathrm{SO}_{8}^{+}(q)$ and the one for the second block of $\mathrm{SO}_{12}^{+}(q)$ have automorphisms induced by the non-trivial graph automorphisms of the underlying groups. But note that also the decomposition matrix for the principal block of $E_{6}$ has an automorphism fixing $\phi_{6,1}, \phi_{80,7}, D_{4}: 2.1$ and $\phi_{6,25}$ and interchanging the other characters in pairs, and similarly, the decomposition matrix for the second block of $E_{7}$ has an automorphism of order two with fixed points $\phi_{280,8}$ and $\phi_{216,16}$.

\section{Decomposition matrices for twisted type Groups}

We now turned to simply-laced groups of twisted type, viz. ${ }^{2} D_{5},{ }^{2} D_{6}$ and ${ }^{2} E_{6}$.

7.1. Decomposition matrices for $\mathrm{SO}_{10}^{-}(q)$. The group $G=\mathrm{SO}_{10}^{-}(q)$ has four unipotent $\ell$-blocks for primes $2 \neq \ell \mid\left(q^{2}+1\right)$, the principal block, one block with cyclic defect and two blocks of defect zero.

Theorem 7.1. Assume that $q$ is odd. Then the $\ell$-modular decomposition matrices for the unipotent blocks of $\mathrm{SO}_{10}^{-}(q)$ of positive defect for primes $\ell$ with $\left(q^{2}+1\right)_{\ell}>5$ are as given in Tables 23 and 24.

Proof. The Brauer tree for the block with cyclic defect is easily seen to be as given in Table 24. We describe how to obtain projectives $\Psi_{1}, \ldots, \Psi_{11}, \Psi_{13}, \Psi_{14}$ for the principal block as in Table 23.

Application of ( $\mathrm{HCi})$ yields $\Psi_{i}$ with $i \in\{1,3,4,5,6,7,8,9,13\}$. Moreover, we obtain $\Psi_{2}+\Psi_{3}, \Psi_{2}+\Psi_{6}$, so that (Sum) gives $\Psi_{2}$. (Alternatively, the projectives in the principal series are obtained from the Iwahori-Hecke algebra $H$ of type $B_{4}$, with parameters $q^{2}$ and q.) Next, (HCi) gives $\tilde{\Psi}_{10}=\Psi_{10}+\Psi_{8}, \tilde{\Psi}_{10}^{\prime}=\Psi_{10}+\Psi_{11}$ and $\tilde{\Psi}_{11}=\Psi_{11}+\Psi_{13}$. Thus, $\tilde{\Psi}_{10}+\tilde{\Psi}_{11}=\tilde{\Psi}_{10}^{\prime}+\Psi_{8}+\Psi_{13}$, which shows that $\Psi_{8}, \Psi_{13}$ occur as summands of $\tilde{\Psi}_{10}+\tilde{\Psi}_{11}$. Nonnegativity of decomposition numbers implies that $\Psi_{8}$ occurs in $\tilde{\Psi}_{10}$ and $\Psi_{11}$ in $\tilde{\Psi}_{11}$, 
TABLE 23. $\mathrm{SO}_{10}^{-}(q), q$ odd, $\left(q^{2}+1\right)_{\ell}>5$

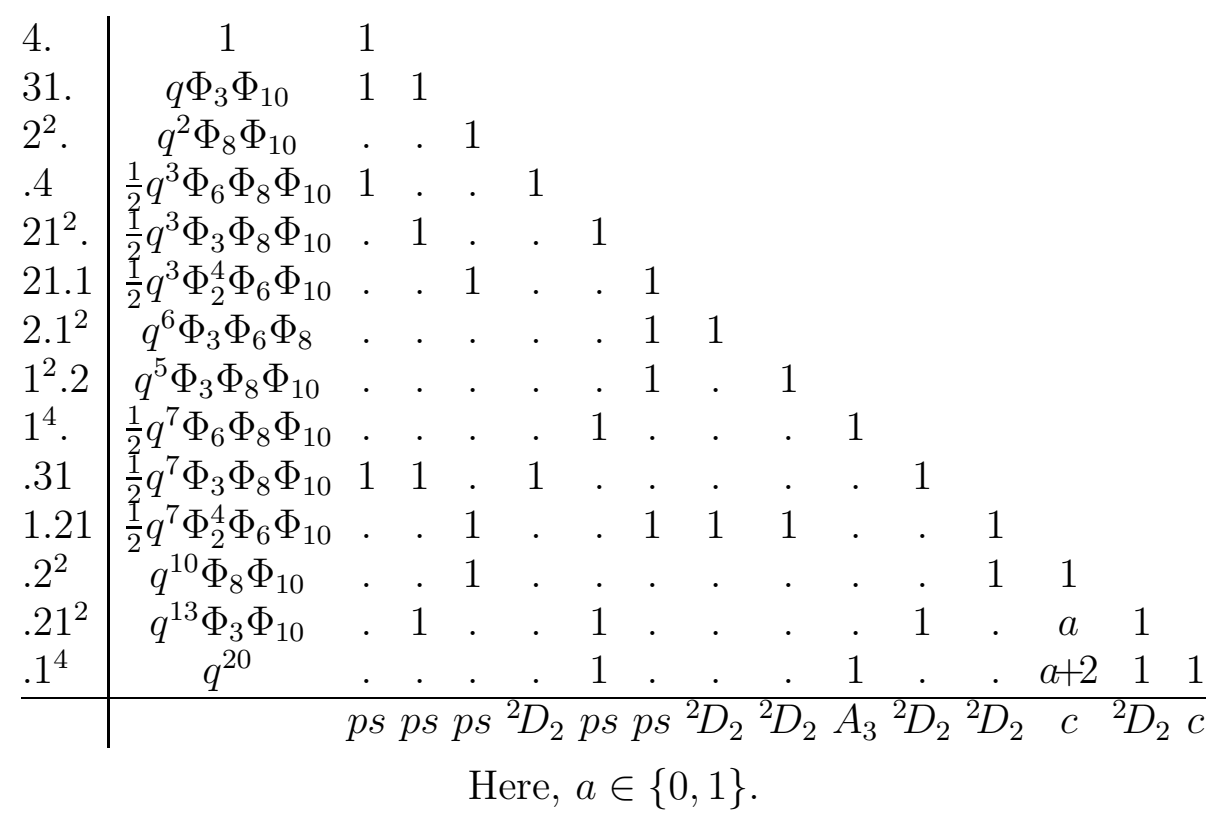

TABLE 24. $\operatorname{SO}_{10}^{-}(q)$, block of defect $1,2 \neq \ell \mid\left(q^{2}+1\right)$

$$
3.1-1.3 \frac{{ }^{2} D_{2}}{{ }^{2} D_{2}} 1.1^{3}-1^{3} .1
$$

so we obtain $\Psi_{10}$ and $\Psi_{11}$. Application of (HCr) also shows that $\Psi_{1}, \ldots, \Psi_{11}$ and $\Psi_{13}$ are indecomposable. The last projective $\Psi_{14}$ is given by (St).

The Hecke algebra for the cuspidal Brauer character of ${ }^{2} D_{2}(q) \cong A_{1}\left(q^{2}\right)$ is of type $B_{3}$, and the parameters are seen locally in the Levi subgroups of types ${ }^{2} D_{4}$ and ${ }^{2} D_{3} \times A_{1}$ to be $q^{4}$ and $q$. From its decomposition matrix it follows that exactly eight simple modules lie in the corresponding Harish-Chandra series in $G$. Since the unipotent block with cyclic defect contains two of them, the principal block will contain the remaining six. We have then accounted for all non-cuspidal Harish-Chandra series, so the remaining Brauer character must all be cuspidal.

By (GGGR), the unipotent part of $\Psi_{12}$ is $\rho_{.2^{2}}+a \rho_{.21^{2}}+b \rho_{.1^{4}}$, where $a$ and $b$ are unknown. To compute $b$ we use (Cox), which gives the relation $b \leq a+2$. The relation $b \geq a+2$ is obtained from the $\ell$-reduction of the non-unipotent character obtained by DeligneLusztig induction of an $\ell$-character in general position of a torus of order $(q+1)\left(q^{2}+1\right)^{2}$ (such a character exists whenever $\left(q^{2}+1\right)_{\ell}>5$ ). Finally, to obtain an upper bound for $a$, we consider the generalized Gelfand-Graev representations of $\mathrm{SO}_{14}^{-}(q)$ associated to the family $\left\{\rho_{1^{5} .1}, \rho_{1^{3} .1^{3}}, \rho_{1.2^{2} 1}, \rho_{.321}\right\}$, and more precisely the one whose projection to this family is $\rho_{.321}+\rho_{1.2^{2} 1}$. The character of this representation, cut by the block containing $\rho_{.321}$ is of the form $\rho_{.321}+\rho_{1.2^{2} 1}+\alpha \rho_{1.1^{5}}$ by (GGGR). The Harish-Chandra restriction of this character yields $a \leq 1$. 
Remark 7.2. If Kawanaka's conjecture (see Remark 3.7) holds for the characters in the family $\left\{\rho_{1^{5} .1}, \rho_{1^{3} .1^{3}}, \rho_{1.2^{2} 1}, \rho_{.321}\right\}$ of $\mathrm{SO}_{14}^{-}(q)$, then the previous argument shows that $a=0$.

Remark 7.3. The 5-modular decomposition matrix of $\mathrm{SO}_{10}^{-}(2)$ is known; there, the last three entries in the partially unknown 12 th column of Table 23 read $(1,0,1)$, whence the case when $\left(q^{2}+1\right)_{\ell}=5$ does behave differently.

7.2. A decomposition matrix for $\mathrm{SO}_{14}^{-}(q)$. The group $G=\mathrm{SO}_{14}^{-}(q)$ has one nonprincipal unipotent $\Phi_{4}$-block of positive defect, which we label by its 4 -Harish-Chandra source in a Levi subgroup of type $\mathrm{SO}_{6}^{-}(q)$.

Theorem 7.4. Assume that $q$ is odd. Then the $\ell$-modular decomposition matrix for the non-principal unipotent block of $\mathrm{SO}_{14}^{-}(q)$ of defect $\Phi_{4}^{2}$, for $\left(q^{2}+1\right)_{\ell}>5$, is as given in Table 25.

TABLE 25. $\mathrm{SO}_{14}^{-}(q), q$ odd, block $\left(\begin{array}{c}013 \\ 1\end{array}\right),\left(q^{2}+1\right)_{\ell}>5$

\begin{tabular}{|c|c|c|c|c|c|c|c|c|c|c|c|c|c|c|}
\hline 5.1 & $\Phi_{3} \Phi_{6}$ & 1 & & & & & & & & & & & & \\
\hline 1.5 & $\frac{1}{2} q^{2} \Phi_{3} \Phi_{8} \Phi_{10} \Phi_{14}$ & 1 & 1 & & & & & & & & & & & \\
\hline 32.1 & $\frac{1}{2} q^{2} \Phi_{3} \Phi_{5} \Phi_{8} \Phi_{14}$ & 1 & . & 1 & & & & & & & & & & \\
\hline 321. & $\frac{1}{2} q^{2} \Phi_{2}^{4} \Phi_{6} \Phi_{10} \Phi_{14}$ & . & . & . & 1 & & & & & & & & & \\
\hline $31^{2} .1$ & $q^{4} \Phi_{3}^{2} \Phi_{6} \Phi_{8} \Phi_{14}$ & . & . & . & 1 & 1 & & & & & & & & \\
\hline $2^{2} 1.1$ & $q^{5} \Phi_{3} \Phi_{5} \Phi_{6} \Phi_{10} \Phi_{14}$ & . & . & 1 & . & . & 1 & & & & & & & \\
\hline $1^{3} .3$ & $q^{7} \Phi_{5} \Phi_{8} \Phi_{10} \Phi_{14}$ & . & . & . & . & 1 & & 1 & & & & & & \\
\hline 1.32 & $q^{9} \Phi_{3} \Phi_{5} \Phi_{6} \Phi_{10} \Phi_{14}$ & 1 & 1 & 1 & . & . & . & . & 1 & & & & & \\
\hline $3.1^{3}$ & $q^{10} \Phi_{5} \Phi_{8} \Phi_{10}$ & . & . & . & . & 1 & . & . & . & 1 & & & & \\
\hline $1.31^{2}$ & $q^{12} \Phi_{3}^{2} \Phi_{6} \Phi_{8} \Phi_{14}$ & . & . & . & 1 & 1 & & 1 & . & 1 & 1 & & & \\
\hline .321 & $\frac{1}{2} q^{14} \Phi_{2}^{4} \Phi_{6} \Phi_{10} \Phi_{14}$ & . & . & . & 1 & . & . & . & . & . & 1 & 1 & & \\
\hline $1.2^{2} 1$ & $\frac{1}{2} q^{14} \Phi_{3} \Phi_{5} \Phi_{8} \Phi_{14}$ & . & . & 1 & & $\cdot$ & 1 & . & 1 & . & . & $a$ & 1 & \\
\hline $1^{5} .1$ & $\frac{1}{2} q^{14} \Phi_{3} \Phi_{8} \Phi_{10} \Phi_{14}$ & . & . & . & . & . & 1 & . & . & . & . & & & 1 \\
\hline $1.1^{5}$ & $q^{28} \Phi_{3} \Phi_{6}$ &. & . & . & . & . & 1 & . & . & . & & $a+2$ & 1 & 1 \\
\hline
\end{tabular}

Here, $a \in\{0,1\}$ is as in Table 23, and all degrees have been divided by $q^{2} \Phi_{4} \Phi_{12}$.

Proof. All columns but the 11 th are obtained by (HCi), as well as $\Psi_{8}+a \Psi_{10}+\Psi_{11}$ and $\Psi_{11}+(1-a) \Psi_{12}$. Thus, independent of the value of $a$ we also recover $\Psi_{11}$ via (Sum).

7.3. Decomposition matrices for ${ }^{2} E_{6}(q)$. We now turn to the $\Phi_{4}$-blocks of the exceptional groups of type ${ }^{2} E_{6}$. There are 10 unipotent $\ell$-blocks of defect zero, one of cyclic defect and the principal block.

Theorem 7.5. Let $(q, 6)=1$. The decomposition matrices for the unipotent $\ell$-blocks of ${ }^{2} E_{6}(q)$ of positive defect, where $\left(q^{2}+1\right)_{\ell}>5$, are as given in Tables [26] and 27. 
TABLE $26 .{ }^{2} E_{6}(q),(q, 6)=1,\left(q^{2}+1\right)_{\ell}>5$

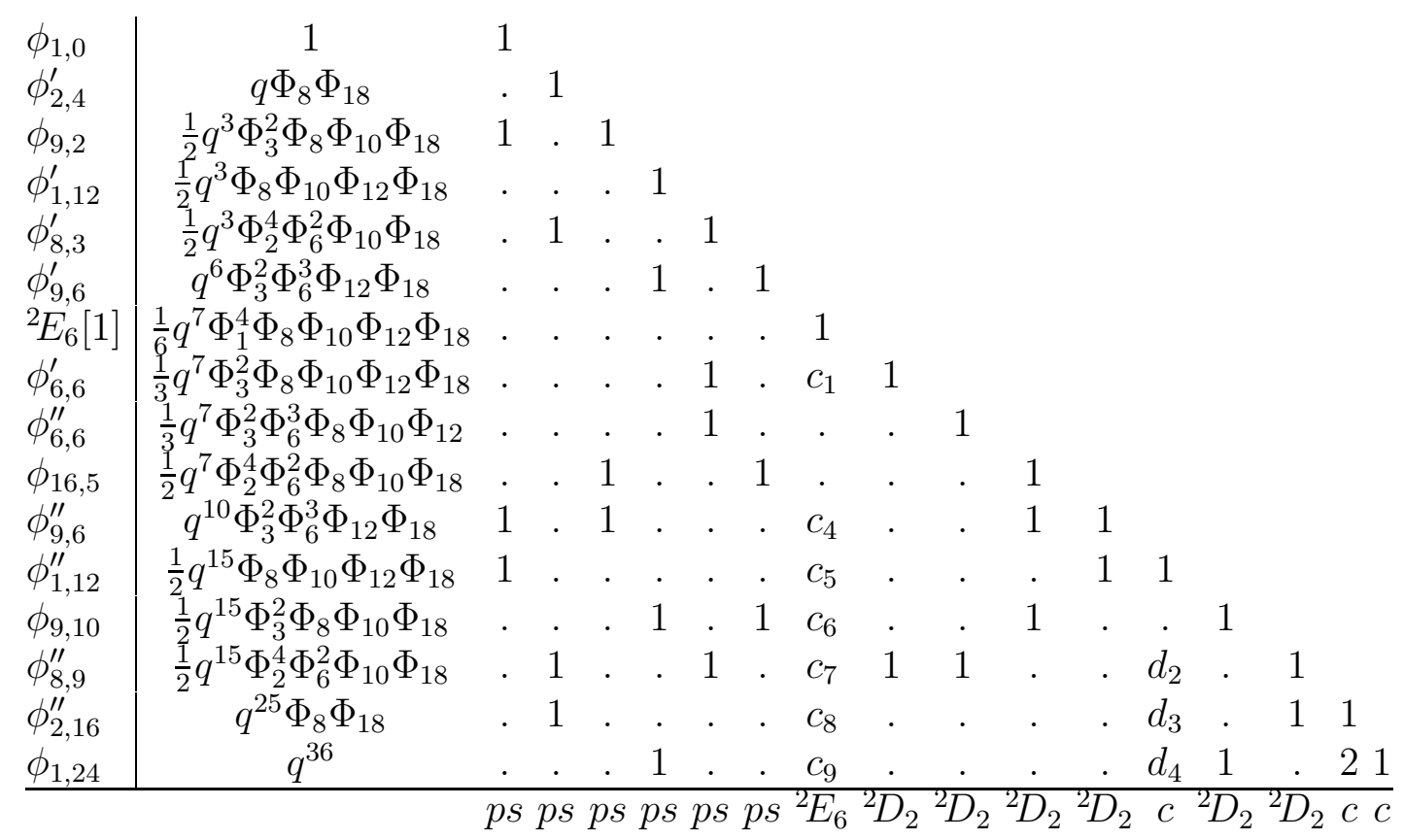

Here, $c_{1} \in\{0,1,2\}, d_{2} \in\{0,1\}, c_{9}=4+2 c_{1}+3 c_{4}-3 c_{5}+c_{6}-2 c_{7}+2 c_{8}$ and $d_{4}=-3-2 d_{2}+2 d_{3}$.

TABLE $27 .{ }^{2} E_{6}(q)$, block of defect $1,2 \neq \ell \mid\left(q^{2}+1\right)$

$$
\phi_{4,1}-\phi_{4,7}^{\prime \prime} \underset{{ }^{2} D_{2}}{-}{ }^{2} D_{2} \quad \phi_{4,13}-\phi_{4,7}^{\prime}
$$

Proof. The Brauer tree for the block with cyclic defect is easily obtained, see also [1, Thm. 3.10]. We now discuss how to find projective characters $\Psi_{i}$, for $i \in\{1-6,8-$ $11,13,14,16\}$, with unipotent parts as given in the columns of Table 26. (HCi) yields $\Psi_{i}$ with $i \in\{1,3,4,6,11,13\}$, which are indecomposable by $(\mathrm{HCr})$. Further, we obtain $\Psi_{2}+\Psi_{4}$ and $\Psi_{2}+\Psi_{3}+\Psi_{6}$, yielding $\Psi_{2}$ by (Sum). Similarly, $\Psi_{5}+2 \Psi_{6}$ and $\Psi_{5}+\Psi_{1}+2 \Psi_{3}$ lead to $\Psi_{5} ; \Psi_{3}+\Psi_{8}+\Psi_{11}$ and $\Psi_{6}+\Psi_{8}$ provide the projective character $\Psi_{8}$, and $\Psi_{3}+\Psi_{9}$ and $\Psi_{6}+\Psi_{9}+\Psi_{13}$ lead to $\Psi_{9}$. Furthermore, $\Psi_{8}+\Psi_{10}$ and $\Psi_{9}+\Psi_{10}$ give $\Psi_{10}$, and $\Psi_{10}+\Psi_{14}$ and $\Psi_{11}+\Psi_{14}$ give rise to $\Psi_{14}$. By inspection using ( $\mathrm{HCr}$ ) all projectives constructed so far are indecomposable.

We claim that we have now accounted for all non-cuspidal Harish-Chandra series. Indeed, the decomposition numbers for the Hecke algebra of type $F_{4}$ with unequal parameters have been calculated in [1, Thm. 3.10] for all $\ell \geq 5$, showing that there are six principal series PIMs. The relative Weyl group of the cuspidal unipotent Brauer character of ${ }^{2} D_{2}(q)=A_{1}\left(q^{2}\right)$ has type $B_{3}$. We have already found all projective indecomposable 
summands in the principal block of the Harish-Chandra induction from proper Levi subgroups in that series, viz. $\Psi_{8}, \Psi_{9}, \Psi_{10}, \Psi_{11}, \Psi_{13}$ and $\Psi_{14}$. Two further PIMs in that series lie in the block of cyclic defect. This accounts for all non-cuspidal Harish-Chandra series. Hence the four missing columns must correspond to cuspidal Brauer characters.

For the remaining columns we consider the following three GGGRs, whose existence is given by [5, Thm. 6.5(ii)], assuming that $p$ is good:

- the GGGR associated to the family containing ${ }^{2} E_{6}[1]$ and with projection ${ }^{2} E_{6}[1]+$ $2 \phi_{6,6}^{\prime}+\phi_{12,4}$ on this family;

- the GGGR associated to the family $\left\{\phi_{2,16}^{\prime}, \phi_{9,10}, \phi_{1,12}^{\prime \prime}, \phi_{8,9}^{\prime \prime}\right\}$ and with projection $\phi_{1,12}^{\prime \prime}+\phi_{8,9}^{\prime \prime}$ on this family;

- the GGGR associated to the family $\left\{\phi_{2,16}^{\prime \prime}\right\}$.

¿From (GGGR) we deduce the unitriangularity of the decomposition matrix. In addition, if we denote by $c_{1}, \ldots, c_{9}$ the unknown entries in the 7th column then $c_{2}=c_{3}=0$ and $c_{1} \leq 2$. Similarly, if $d_{1}, \ldots, d_{4}$ denote the entries in the 12 th column then $d_{1}=0$ and $d_{2} \leq 1$. The last unknown entry (in the 15 th column) will be denoted by $d_{5}$.

A Sylow $\Phi_{4}$-torus of $G$ has a regular $\ell$-character $\theta$ whenever $\left(q^{2}+1\right)_{\ell}>5$. By (Red), the $\ell$-reduction of a non-unipotent character induced from $\theta$ yields relations on the $c_{i}$ 's and $d_{i}$ 's, namely $d_{5} \geq 2,3+2 d_{2}-2 d_{3}+d_{4} \geq 0$ and $-4-2 c_{1}-3 c_{4}+3 c_{5}-c_{6}+2 c_{7}-2 c_{8}+c_{9} \geq 0$. To obtain the opposite inequalities we use (DL) successively for the elements $s_{1} s_{2} s_{3} s_{4}$, $s_{1} s_{2} s_{3} s_{1} s_{4} s_{3}$ and $s_{1} s_{2} s_{4} s_{3} s_{1} s_{5} s_{4} s_{3} s_{6} s_{5} s_{4} s_{3}$.

As in Proposition 3.8, we can use [8, Conj. 1.2] to obtained conjectural upper bounds on the unknown entries in the decomposition matrix which do not depend on $q$.

Proposition 7.6. Assume Conjecture 1.2 in [8] holds. Then in the decomposition matrix of the principal $\Phi_{4}$-block of ${ }^{2} E_{6}(q)$, we have $c_{1}=0, c_{4} \leq 3, c_{5} \leq 26, c_{6} \leq 29, c_{7} \leq 50$, $c_{8} \leq 156$ and $d_{3} \leq 6$.

Proof. We consider virtual characters $Q_{w}$ afforded by the Alvis-Curtis dual of the intersection cohomology of suitably chosen Deligne-Lusztig varieties. In the following table, we give, for each element $w$ we consider, the multiplicity of a PIM $\Psi_{i}$ in $Q_{w}[\lambda]$. In order to simplify notation, we denote $s_{1}, \ldots, s_{6}$ by $1, \ldots, 6$.

\begin{tabular}{r|c|c|l}
$w$ & $\lambda$ & $i$ & $\left\langle Q_{w}[\lambda], \varphi_{i}\right\rangle$ \\
\hline 1231454236542314356 & 1 & 8 & $-36 c_{1}$ \\
123423145431 & 1 & 11 & $3-c_{4}$ \\
& & 12 & $23+c_{4}-c_{5}$ \\
2342314354316543 & 1 & 13 & $6\left(29-c_{6}\right)$ \\
23143154316543 & 1 & 14 & $6\left(50+c_{1}-d_{2}\left(23+c_{4}-c_{5}\right)-c_{7}\right)$ \\
546542 & -1 & 15 & $2\left(106-c_{1}+c_{7}+\left(d_{2}-d_{3}\right)\left(23+c_{4}-c_{5}\right)-c_{8}\right)$ \\
& & & $5+d_{2}-d_{3}$
\end{tabular}

Conjecture 1.2 in [8] predicts that the entries in the last column of the previous table are non-negative. We deduce that $c_{1}=0, c_{4} \leq 3, c_{5} \leq c_{4}+23 \leq 26, c_{6} \leq 29, c_{7} \leq$ 
$50+c_{1}-d_{2}\left(23+c_{4}-c_{5}\right) \leq 50$,

$c_{8} \leq 106-c_{1}+c_{7}+\left(d_{2}-d_{3}\right)\left(23+c_{4}-c_{5}\right) \leq 106-c_{1}+c_{7}+d_{2}\left(23+c_{4}-c_{5}\right) \leq 156$

and $d_{3} \leq d_{2}+5 \leq 6$ since $d_{2} \in\{0,1\}$.

7.4. The $\Phi_{4}$-blocks in twisted groups. As in the untwisted case we collect some data on the $\Phi_{4}$-blocks studied above in Table 28 .

TABLE 28. HC-series in $\Phi_{4}$-blocks of twisted groups

\begin{tabular}{r|cc|cccccc}
$G$ & $W_{G}(b)$ & $|\operatorname{IBr}(b)|$ & $p s$ & ${ }^{2} D_{2}$ & $A_{3}$ & $.2^{2}$ & $.1^{4}$ & $c$ \\
\hline${ }^{2} D_{5}$ & $G(4,1,2)$ & 14 & 5 & 6 & 1 & 1 & 1 & \\
${ }^{2} D_{7}$ & & & 5 & 6 & 1 & 1 & 1 & \\
\hline${ }^{2} E_{6}$ & $G_{8}$ & 16 & 6 & 6 & & & & 4
\end{tabular}

Note that the decomposition matrices for the blocks in ${ }^{2} D_{5}$ and in ${ }^{2} D_{7}$ of defect $\Phi_{4}^{2}$ coincide after permuting rows and columns suitably. Again, it would be interesting to see whether this is caused by a Morita equivalence between these blocks. On the other hand, the multisets of entries of the matrices for twisted groups differ from those for any of the untwisted ones, so if there exits a Morita equivalence between blocks for twisted and untwisted groups, it would have to be with respect to a different choice of basic sets.

\section{Decomposition matrices For SymplectiC Groups And $F_{4}(q)$}

We now turn to groups with non-simply laced Dynkin diagram, where we start by giving (approximations) to decomposition matrices for the unipotent blocks of small rank symplectic groups $\operatorname{Sp}_{2 n}(q)$ for primes $\ell \mid\left(q^{2}+1\right)$. Again, it is not known a priori in our present situation that the decomposition matrix has triangular shape. This leads to additional complications.

For completeness and for use in the subsequent proofs, we recall the known Brauer trees for $\operatorname{Sp}_{4}(q)$ and $\operatorname{Sp}_{6}(q)$ (see [10]). We also indicate the modular Harish-Chandra series of the PIMs.

Theorem 8.1 (Fong-Srinivasan). Let $2 \neq \ell \mid\left(q^{2}+1\right)$ be a prime. Then the Brauer trees for the unipotent $\ell$-blocks of $\operatorname{Sp}_{4}(q)$ and $\operatorname{Sp}_{6}(q)$ are as given in Table 29 .

Next, let $G=\operatorname{Sp}_{8}(q)$.

Theorem 8.2. Let $\ell$ be a prime. Assume that $q$ is odd. Then the decomposition matrices for the unipotent $\ell-$ blocks of $\operatorname{Sp}_{8}(q),\left(q^{2}+1\right)_{\ell}>5$, are as given in Tables 30 and 31 .

Proof. The group $G=\operatorname{Sp}_{8}(q)$ has three unipotent blocks of $\Phi_{4}$-defect zero, two blocks of $\Phi_{4}$-defect 1 with four characters each, and all other unipotent characters lie in the principal block. The Brauer trees for the blocks of defect 1 are known by [10] and in any case can easily be recovered by $(\mathrm{HCi})$.

The projective modules $\Psi_{i}$ for $i \in\{1, . ., 9,11,13\}$ are obtained by (HCi). Using (HCr), we can check that there are indecomposable. Finally, (St) yields the last column, leaving 
TABLE 29. $\operatorname{Sp}_{4}(q)$ and $\operatorname{Sp}_{6}(q), 2 \neq \ell \mid\left(q^{2}+1\right)$

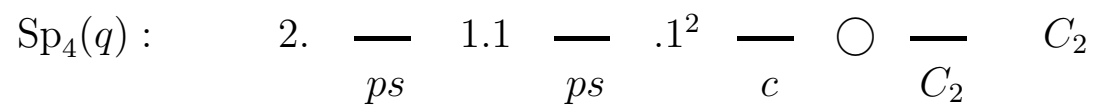

$$
\begin{aligned}
& \operatorname{Sp}_{6}(q): \quad 3 .-1.2-.21-\bigcirc-C_{2}: 1^{2} \\
& \text { 21. } \underset{p s}{-} 1^{2} .1 \underset{\text { ps }}{-} .^{3} \underset{.1^{2}}{-} \bigcirc \underset{C_{2}}{-} C_{2}: 2
\end{aligned}
$$

TABLE 30. $\operatorname{Sp}_{8}(q),\left(q^{2}+1\right)_{\ell}>5$

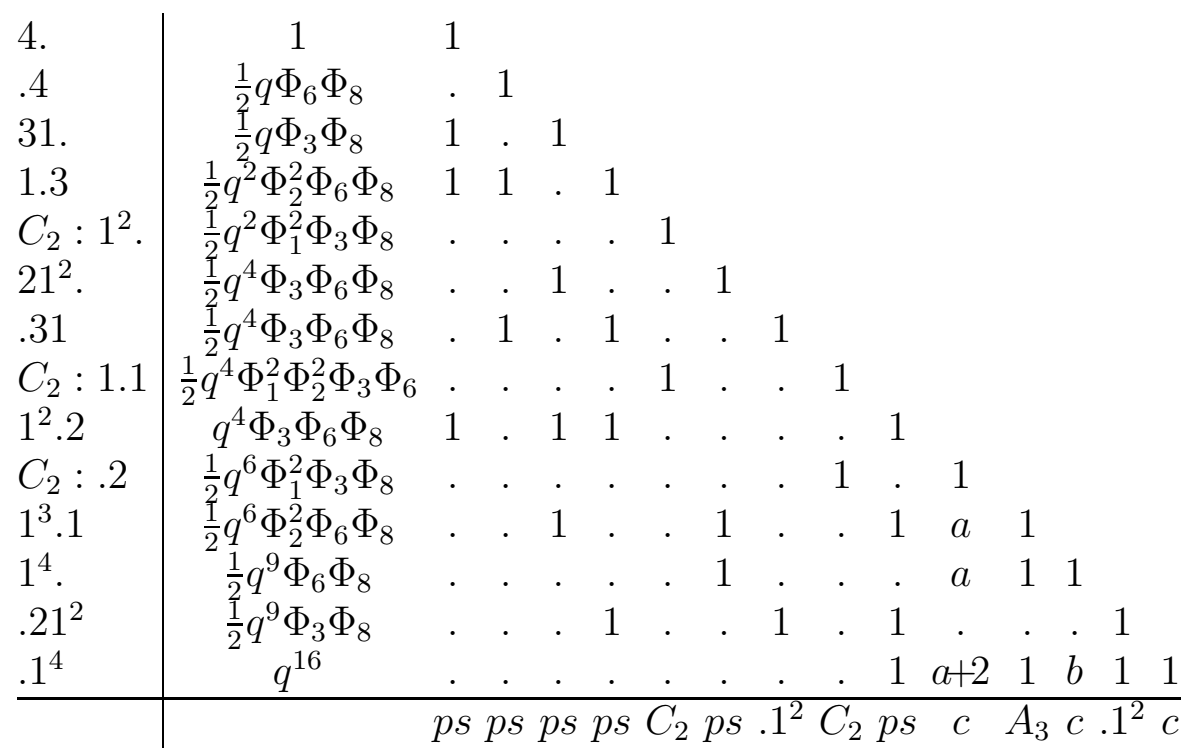

Here, $a \in\{0,1\}$ and $b \in\{0,1,2,3\}$.

TABLE $31 . \operatorname{Sp}_{8}(q)$, blocks of defect $1,2 \neq \ell \mid\left(q^{2}+1\right)$

$$
\begin{aligned}
& 3.1-2.2-.2^{2}-\bigcirc-C_{2}: 1^{2} \\
& 2^{2} \cdot{ }_{p s} 1^{2} \cdot 1^{2}-1.1^{3} \frac{-}{.1^{2}} \bigcirc \frac{-}{C_{2}} C_{2}: 2 .
\end{aligned}
$$

only two (necessarily cuspidal) PIMs to determine. A first approximation of these columns is given by (GGGR) for the families $\left\{\rho_{1^{3} .1}, \rho_{1^{2} .1^{2}}, \rho_{.2^{2}}, \rho_{C_{2}: .2}\right\}$ and $\left\{\rho_{1^{4}}, \rho_{1.1^{3}}, \rho_{.21^{2}}, \rho_{C_{2}: 1^{2}}\right\}$. 
We deduce that the relevant submatrix for the last five projectives now has the form

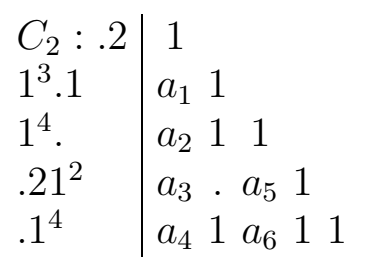

In addition, [5, Thm. 6.5(ii)] yields $a_{1} \in\{0,1\}$ and $a_{5}=0$.

As usual, relations on the $a_{i}$ 's are obtained by looking at suitable Deligne-Lusztig characters: from (Cox) we deduce that $a_{1}-a_{2} \geq 0$ and $a_{1}+a_{3}-a_{4}+2 \geq a_{6}\left(a_{1}-a_{2}\right)$. But from (Red) with the induction of an $\ell$-character of a Sylow $\Phi_{4}$-torus in general position, which exists whenever $\left(q^{2}+1\right)_{\ell}>5$, we get $a_{1}+a_{3}-a_{4}+2 \leq a_{2}-a_{1}$. Consequently, $0 \leq a_{6}\left(a_{1}-a_{2}\right) \leq a_{2}-a_{1} \leq 0$ which forces $a_{1}=a_{2}$ and $a_{4}=a_{1}+a_{3}+2$. With (DL) applied to the element $w=s_{1} s_{2} s_{3} s_{2} s_{1} s_{2} s_{3} s_{4}$ of the Weyl group we obtain $a_{6} \leq 3$.

Finally, we use the GGGR of $\operatorname{Sp}_{10}(q)$ associated to the family $\left\{\rho_{1^{4} .1}, \rho_{1^{2} .1^{3}}, \rho_{.2^{2} 1}, \rho_{C_{2}: .21}\right\}$ with projection $\rho_{C_{2}: .21}+\rho_{1^{4} .1}$ to this family. Cut by the block containing these two characters, the unipotent part of this GGGR is of the form $\rho_{C_{2}: 21}+\rho_{1^{4} .1}+\alpha \rho_{.1^{5}}$. Its Harish-Chandra restriction to $G$ forces $a_{3}=0$. Setting $a=a_{1}$ and $b=a_{6}$, we obtain the decomposition matrix as shown in Table 30

Remark 8.3. Kawanaka's conjecture [17, Conj. 2.4.5] would imply $a=0$ (see also Remark 3.7).

Theorem 8.4. Let $\ell$ be a prime. The decomposition matrix for the principal $\ell$-block of $F_{4}(q),(q, 6)=1,\left(q^{2}+1\right)_{\ell}>5$, is as given in Table 32.

For the unipotent blocks with cyclic defect, see [18, Lemma 5.4].

Proof. We start from the approximation to the decomposition matrix which was obtained in the thesis of Köhler [18. The relevant submatrix for the last eight projectives has the following form:

$$
\begin{array}{l|cccccccc}
F_{4}^{I I}[1] & 1 & & & & & & & \\
F_{4}^{I}[1] & \cdot & 1 & & & & & & \\
F_{4}[-I] & \cdot & \cdot & 1 & & & & & \\
F_{4}[I] & \cdot & \cdot & \cdot & 1 & & & & \\
\phi_{9,10} & \cdot & \cdot & c_{1} & c_{1} & 1 & & & \\
\phi_{4,13} & a_{1} & . & c_{2} & c_{2} & 1 & 1 & & \\
B_{2}: 1^{2} & \cdot & b_{1} & c_{3} & c_{3} & \cdot & . & 1 & \\
\phi_{1,24} & a_{2} & b_{2} & c_{4} & c_{4} & 1 & d & e & 1
\end{array}
$$

First relations come from the $\ell$-reduction of non-unipotent characters. For each uniform character $\rho \in \mathcal{E}(G,(s))$ we construct, we give in the table below the type of $C_{\mathbf{G}}(s)$, the Jordan correspondent $\rho_{s}$ of $\rho$ and the relations that we will use:

\begin{tabular}{c|c|c}
$C_{\mathbf{G}}(s)$ & $\rho_{s}$ & relations \\
\hline$B_{2} \cdot\left(q^{2}+1\right)$ & $\rho_{1^{2}}+\rho_{B_{2}}$ & $c_{2} \geq c_{1}-1$ \\
\hline$\left(q^{2}+1\right)^{2}$ & 1 & $b_{2} \geq 2 b_{1}-3$ \\
& & $c_{4} \geq 3 c_{1}-2 c_{2}+2 c_{3}-4$ \\
& & $e \geq 2$
\end{tabular}


TABLE $32 . F_{4}(q),(q, 6)=1,\left(q^{2}+1\right)_{\ell}>5$

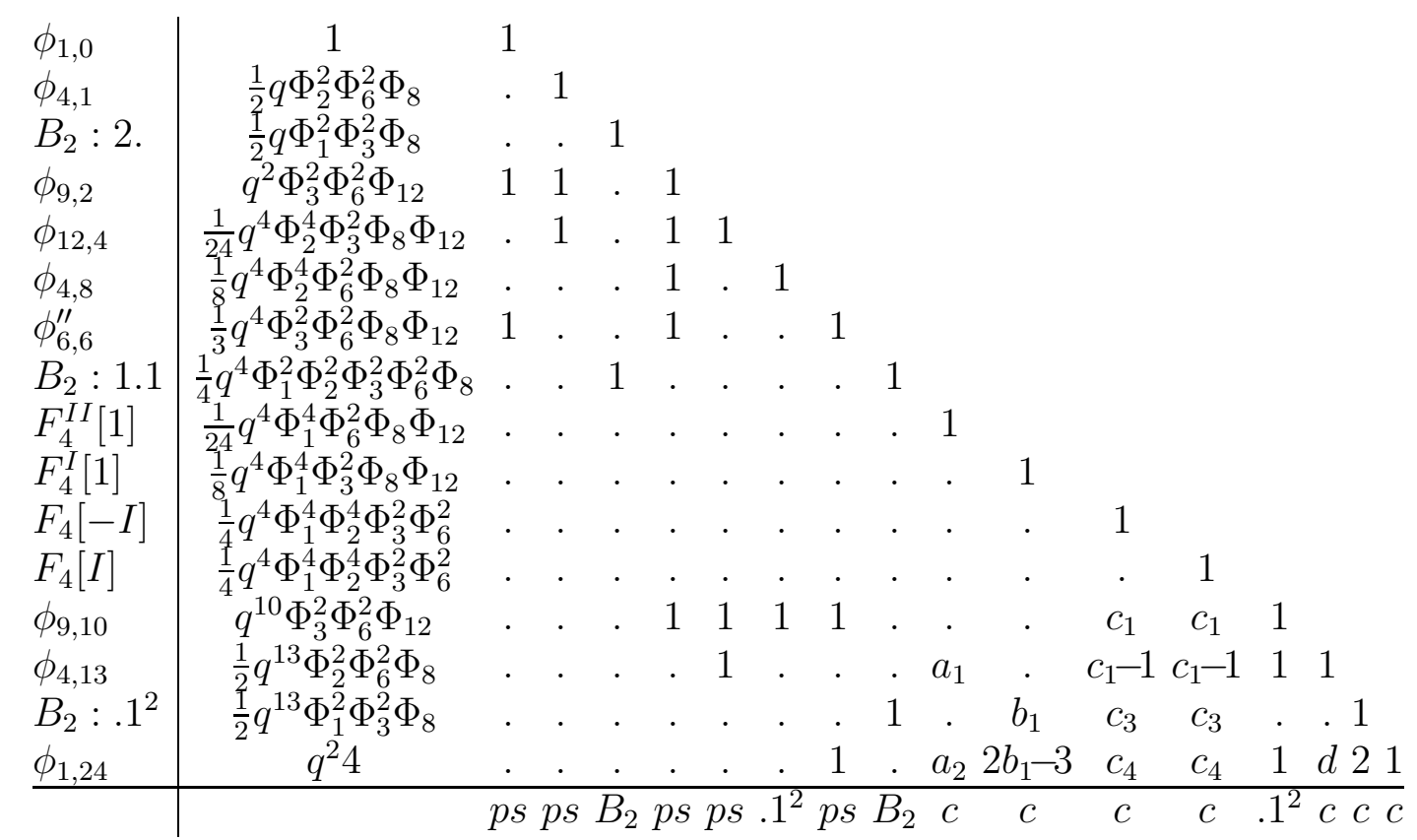

Here, $a_{1} \leq 5, a_{2} \leq 13+\left(5-a_{1}\right) d, c_{3} \in\{0,1\}, c_{4}=c_{1}+2 c_{3}-2$ and $d \in\{0,1,2\}$.

Now we apply (DL) successively to obtain relations on the unknown entries. Starting with the Deligne-Lusztig character associated with a Coxeter element $w$ we find the nonnegative coefficients $c_{1}-c_{2}-1,3-2 c_{3}$ and $2+2 c_{1}-2 c_{4}-3 e+2 c_{3} e$ in $R_{w}$ of the PIMs corresponding to columns $9,10,14$ and 16 , so that $c_{2}=c_{1}-1$ and $c_{3} \in\{0,1\}$. The second relation can then be written $-2\left(4-3 c_{1}+2 c_{2}-2 c_{3}+c_{4}\right)+\left(3-2 c_{3}\right)(2-e) \geq 0$. Since it is a sum of two nonpositive integers, we obtain $4-3 c_{1}+2 c_{2}-2 c_{3}+c_{4}=0$ and $\left(3-2 c_{3}\right)(2-e)=0$, so that $c_{4}=c_{1}+2 c_{3}-2$ and $e=2$. Consequently, the PIMs corresponding to the columns $9,10,14$ and 16 do not occur in $R_{w}$.

We continue with the Deligne-Lusztig character associated with $w^{\prime}=s_{1} s_{2} s_{3} s_{4} s_{1} s_{2} s_{3} s_{4}$. Using (DL) we find $2 b_{1}-b_{2}-3 \geq 0$ which forces $b_{2}=2 b_{1}-3$ by the previous inequalities. In addition, the PIMs corresponding to the columns 9, 14 and 16 still do not occur in $R_{w^{\prime}}$.

Finally, with $w^{\prime \prime}=s_{1} s_{2} s_{3} s_{4} s_{1} s_{2} s_{3} s_{4} s_{1} s_{2} s_{3} s_{4}$ we consider the characters $R_{w^{\prime \prime}}[\lambda]$ for various eigenvalues $\lambda$ of $F$. The multiplicities of the 14th and 16th PIM in these virtual characters yield the relations

$$
a_{1} \leq 5, \quad d \leq 2, \quad a_{2} \leq 13+\left(a_{1}-5\right) d
$$

(in particular $a_{2} \leq 13$ ).

Up to finitely many possibilities, the decomposition matrix given above depends only on two unknown parameters, viz. $b_{1}, c_{1}$. Moreover, these could be bounded above by suitable polynomials in $q$ using GGGRs. As in Proposition 3.8, we can also produce conjectural bounds independent of $q$. 
Proposition 8.5. Assume Conjecture 1.2 in [8] holds. Then in the decomposition matrix of the principal $\Phi_{4}$-block of $F_{4}(q)$ we have $b_{1} \in\{2,3,4\}$ and $c_{1} \in\{1,2\}$.

Proof. We compute the Alvis-Curtis dual of the intersection cohomology of two DeligneLusztig varieties, corresponding to the elements $w_{1}=s_{1} s_{2} s_{3} s_{4}$ and $w_{2}=s_{2} s_{4} s_{3} s_{2} s_{1} s_{3} s_{2} s_{3}$. Conjecture 1.2 in [8] predicts that the corresponding characters, denoted in [8] by $Q_{w_{1}}$ and $Q_{w_{2}}$, are, up to sign, the unipotent part of projective characters. The same holds for the generalized eigenspaces of $F$ on these characters. The multiplicity of $\Psi_{13}$ in $Q_{w_{1}}$ is $5-2 c_{1}$, which forces $c_{1} \leq 2$, and the multiplicity of $\Psi_{15}$ in the 1-eigenspace of $F$ on $Q_{w_{2}}$ is $4-b_{1}$, which forces $b_{1} \leq 4$.

We collect information on the Harish-Chandra series for the blocks considered in this section in the subsequent Table 33 .

TABLE 33. HC-series in $\Phi_{4}$-blocks of defect $\Phi_{4}^{2}$

\begin{tabular}{r|cc|ccccccc}
$G$ & $W_{G}(b)$ & $|\operatorname{IBr}(b)|$ & ps & $B_{2}$ & $.1^{2}$ & $C_{2}$ & $.1^{2}$ & $A_{3}$ & $c$ \\
\hline$B_{4}$ & $G(4,1,2)$ & 14 & 6 & 2 & 2 & & & 1 & 3 \\
$C_{4}$ & & & 6 & & & 2 & 2 & 1 & 3 \\
\hline$F_{4}$ & $G_{8}$ & 16 & 5 & 1 & 1 & 1 & 1 & & 7
\end{tabular}

\section{REFERENCES}

[1] K. Bremke, The decomposition numbers of Hecke algebras of type $F_{4}$ with unequal parameters. Manuscripta Math. 83 (1994), 331-346.

[2] M. Broué, Isométries de caractères et équivalences de Morita ou dérivées. Inst. Hautes Études Sci. Publ. Math. No. 71 (1990), 45-63.

[3] M. Broué, G. Malle, J. Michel, Generic blocks of finite reductive groups. Astérisque 212 (1993), 7-92.

[4] P. Deligne and G. Lusztig, Representations of reductive groups over finite fields. Ann. of Math. 103 (1976), 103-161.

[5] F. Digne, G. Lehrer, J. Michel, On character sheaves and characters of reductive groups at unipotent classes. Preprint, arXiv:1307.0698, 2013.

[6] R. Dipper, On quotient of Hom-functors and representations of finite general linear groups I. J. Algebra 130 (1990), 235-259.

[7] O. Dudas, G. Malle, Decomposition matrices for unitary groups. Submitted, 2013.

[8] O. Dudas, G. Malle, Projective modules in the intersection cohomology of Deligne-Lusztig varieties. C. R. Acad. Sci. Paris Sér. I Math. 352 (2014), 467-471.

[9] O. DudAs, A note on decomposition numbers for groups of Lie type of small rank. J. Algebra 388 (2013), 364-373.

[10] P. Fong, B. SRinivasan, Brauer trees in classical groups. J. Algebra 131 (1990), 179-225.

[11] M. GECK, Verallgemeinerte Gelfand-Graev Charaktere und Zerlegungszahlen endlicher Gruppen vom Lie-Typ. Dissertation, RWTH Aachen, 1990.

[12] M. Geck, G. Hiss, Modular representations of finite groups of Lie type in non-defining characteristic. In: Finite reductive groups: related structures and representations (ed. M. Cabanes), pp. 195-249, Birkhäuser, Basel, 1997.

[13] M. Geck, G. Hiss, G. Malle, Cuspidal unipotent Brauer characters. J. Algebra 168 (1994), $182-220$. 
[14] M. GECK, N. JACON, Representations of Hecke algebras at roots of unity. Algebra and Applications, 15. Springer-Verlag, London, 2011.

[15] M. GECK, J. MÜLLER, James' conjecture for Hecke algebras of exceptional type. I. J. Algebra 321 (2009), 3274-3298.

[16] M. Geck, G. Pfeiffer, Unipotent characters of the Chevalley groups $D_{4}(q)$, $q$ odd. Manuscripta Math. 76 (1992), 281-304.

[17] N. Kawanaka, Shintani Lifting and Gelfand-Graev Representations. The Arcata Conference on Representations of Finite Groups (Arcata, Calif., 1986), 147-163, Proc. Sympos. Pure Math., 47, Part 1. Amer. Math. Soc., Providence, RI, 1987.

[18] C. KöHLER, Unipotente Charaktere und Zerlegungszahlen der endlichen Chevalleygruppen vom Typ $F_{4}$. Dissertation, RWTH Aachen, 2006.

[19] G. Lusztig, Characters of reductive groups over a finite field. Annals of Mathematics Studies, 107. Princeton University Press, Princeton, NJ, 1984.

[20] G. Lusztig, A unipotent support for irreducible representations. Adv. Math. 94 (1992), 139-179.

[21] J. MicheL, The development version of the CHEVIE package of GAP3. Preprint, arXiv: 1310.7905, 2013.

[22] H. Miyachi, Rouquier blocks in Chevalley groups of type E. Adv. Math. 217 (2008), 2841-2871.

[23] J. TAYLOR, Generalised Gelfand-Graev representations in small characteristics. Preprint, arXiv: $1408.1643,2014$.

Université Paris Diderot, UfR de Mathématiques, Bâtiment Sophie Germain, 5 rue Thomas Mann, 75205 Paris CEDEX 13, France.

E-mail address: olivier.dudas@imj-prg.fr

FB Mathematik, TU Kaiserslautern, Postfach 3049, 67653 Kaiserslautern, Germany.

E-mail address: malle@mathematik.uni-kl.de 